\title{
The Generalized Distributive Law
}

\author{
Srinivas M. Aji and Robert J. McEliece, Fellow, IEEE
}

\begin{abstract}
In this semitutorial paper we discuss a general message passing algorithm, which we call the generalized distributive law (GDL). The GDL is a synthesis of the work of many authors in the information theory, digital communications, signal processing, statistics, and artificial intelligence communities. It includes as special cases the Baum-Welch algorithm, the fast Fourier transform (FFT) on any finite Abelian group, the Gallager-Tanner-Wiberg decoding algorithm, Viterbi's algorithm, the BCJR algorithm, Pearl's "belief propagation" algorithm, the Shafer-Shenoy probability propagation algorithm, and the turbo decoding algorithm. Although this algorithm is guaranteed to give exact answers only in certain cases (the "junction tree" condition), unfortunately not including the cases of GTW with cycles or turbo decoding, there is much experimental evidence, and a few theorems, suggesting that it often works approximately even when it is not supposed to.
\end{abstract}

Index Terms-Belief propagation, distributive law, graphical models, junction trees, turbo codes.

\section{INTRODUCTION}

$\mathbf{T}$ HE humble distributive law, in its simplest form, states that $a b+a c=a(b+c)$. The left side of this equation involves three arithmetic operations (one addition and two multiplications), whereas the right side needs only two. Thus the distributive law gives us a "fast algorithm" for computing $a b+a c$. The object of this paper is to demonstrate that the distributive law can be vastly generalized, and that this generalization leads to a large family of fast algorithms, including Viterbi's algorithm and the fast Fourier transform (FFT).To give a better idea of the potential power of the distributive law and to introduce the viewpoint we shall take in this paper, we offer the following example.

Example 1.1: Let $f(x, y, w)$ and $g(x, z)$ be given real-valued functions, where $x, y, z$, and $w$ are variables taking values in a finite set $A$ with $q$ elements. Suppose we are given the task of computing tables of the values of $\alpha(x, w)$ and $\beta(y)$, defined as follows:

$$
\begin{aligned}
\alpha(x, w) & \stackrel{\text { def }}{=} \sum_{y, z \in A} f(x, y, w) g(x, z) \\
\beta(y) & \stackrel{\text { def }}{=} \sum_{x, z, w \in A} f(x, y, w) g(x, z) .
\end{aligned}
$$

Manuscript received July 8, 1998; revised September 23, 1999. This work was supported by NSF under Grant NCR-9505975, AFOSR under Grant 5F49620-97-1-0313, and a Grant from Qualcomm. A portion of McEliece's contribution was performed at the Sony Corporation in Tokyo, Japan, while he was a holder of a Sony Sabbatical Chair. Preliminary versions of this paper were presented at the IEEE International Symposium on Information Theory, Ulm, Germany, June 1997, and at ISCTA 1997, Ambleside U.K., July 1997.

The authors are with the Department of Electrical Engineering, California Institute of Technology, Pasadena, CA 91125 USA (e-mail: \{mas; rjm \} @ systems.caltech.edu).

Communicated by F. R. Kschischang, Associate Editor for Coding Theory. Publisher Item Identifier S 0018-9448(00)01679-5.
(We summarize (1.1) by saying that $\alpha(x, w)$ is obtained by "marginalizing out" the variables $y$ and $z$ from the function $f(x, y, w) g(x, z)$. Similarly, $\beta(y)$ is obtained by marginalizing out $x, z$, and $w$ from the same function.) How many arithmetic operations (additions and multiplications) are required for this task? If we proceed in the obvious way, we notice that for each of the $q^{2}$ values of $(x, w)$ there are $q^{2}$ terms in the sum defining $\alpha(x, w)$, each term requiring one addition and one multiplication, so that the total number of arithmetic operations required for the computation of $\alpha(x, w)$ is $2 q^{4}$. Similarly, computing $\beta(y)$ requires $2 q^{4}$ operations, so computing both $\alpha(x, w)$ and $\beta(y)$ using the direct method requires $4 q^{4}$ operations.

On the other hand, because of the distributive law, the sum in (1.1) factors

$$
\alpha(x, w)=\left(\sum_{y \in A} f(x, y, w)\right) \cdot\left(\sum_{z \in A} g(x, z)\right) .
$$

Using this fact, we can simplify the computation of $\alpha(x, w)$. First we compute tables of the functions $\alpha_{1}(x, w)$ and $\alpha_{2}(x)$ defined by

$$
\begin{gathered}
\alpha_{1}(x, w) \stackrel{\text { def }}{=} \sum_{y \in A} f(x, y, w) \\
\alpha_{2}(x) \stackrel{\text { def }}{=} \sum_{z \in A} g(x, z),
\end{gathered}
$$

which requires a total of $q^{3}+q^{2}$ additions. Then we compute the $q^{2}$ values of $\alpha(x, w)$ using the formula (cf. (1.3))

$$
\alpha(x, w)=\alpha_{1}(x, w) \alpha_{2}(x)
$$

which requires $q^{2}$ multiplications. Thus by exploiting the distributive law, we can reduce the total number of operations required to compute $\alpha(x, w)$ from $2 q^{4}$ to $q^{3}+2 q^{2}$. Similarly, the distributive law tells us that (1.2) can be written as

$$
\begin{aligned}
\beta(y) & =\sum_{x, w \in A} f(x, y, w)\left(\sum_{z \in A} g(x, z)\right) \\
& =\sum_{x, w \in A} f(x, y, w) \alpha_{2}(x)
\end{aligned}
$$

where $\alpha_{2}(x)$ is as defined in (1.4). Thus if we precompute a table of the values of $\alpha_{2}(x)$ ( $q^{2}$ operations), and then use (1.6) ( $2 q^{3}$ further operations), we only need $2 q^{3}+q^{2}$ operations (as compared to $2 q^{4}$ for the direct method) to compute the values of $\beta(y)$.

Finally, we observe that to compute both $\alpha(x, w)$ and $\beta(y)$ using the simplifications afforded by (1.3) and (1.6), we only need to compute $\alpha_{2}(x)$ once, which means that we can compute 
the values of $\alpha(x, w)$ and $\beta(y)$ with a total of only $3 q^{3}+2 q^{2}$ operations, as compared to $4 q^{4}$ for the direct method.

The simplification in Example 1.1 was easy to accomplish, and the gains were relatively modest. In more complicated cases, it can be much harder to see the best way to reorganize the calculations, but the computational savings can be dramatic. It is the object of this paper to show that problems of the type described in Example 1.1 have a wide range of applicability, and to describe a general procedure, which we called the generalized distributive law (GDL), for solving them efficiently. Roughly speaking, the GDL accomplishes its goal by passing messages in a communications network whose underlying graph is a tree.

Important special cases of the GDL have appeared many times previously. In this paper, for example, we will demonstrate that the GDL includes as special cases the fast Hadamard transform, Viterbi's algorithm, the BCJR algorithm, the Gallager-Tanner-Wiberg decoding algorithm (when the underlying graph is cycle-free), and certain "probability propagation" algorithms known in the artificial intelligence community. With a little more work, we could have added the FFT on any finite Abelian group, the Baum-Welch "forward-backward" algorithm, and discrete-state Kalman filtering. Although this paper contains relatively little that is essentially new (for example, the 1990 paper of Shafer and Shenoy [33] describes an algorithm similar to the one we present in Section III), we believe it is worthwhile to present a simply stated algorithm of such wide applicability, which gives a unified treatment of a great many algorithms whose relationship to each other was not fully understood, if sensed at all.

Here is an outline of the paper. In Section II, we will state a general computational problem we call the MPF ("marginalize a product function") problem, and show by example that a number of classical problems are instances of it. These problems include computing the discrete Hadamard transform, maximum-likelihood decoding of a linear code over a memoryless channel, probabilistic inference in Bayesian networks, a "probabilistic state machine" problem, and matrix chain multiplication. In Section III, we shall give an exact algorithm for solving the MPF problem (the GDL) which often gives an efficient solution to the MPF problem. In Section IV we will discuss the problem of finding junction trees (the formal name for the GDL's communication network), and "solve" the example instances of the MPF problem given in Section II, thereby deriving, among other things, the fast Hadamard transform and Viterbi's algorithm. In Section V we will discuss the computational complexity of the GDL. (A proof of the correctness of the GDL is given in the Appendix.)

In Section VI, we give a brief history of the GDL. Finally, in Section VII, we speculate on the possible existence of an efficient class of approximate, iterative, algorithms for solving the MPF problem, obtained by allowing the communication network to have cycles. This speculation is based partly on the fact that two experimentally successful decoding algorithms, viz., the GTW algorithm for low-density parity-check codes, and the turbo decoding algorithm, can be viewed as an application of the GDL methodology on networks with cycles, and partly on some recent theoretical work on GDL-like algorithms on graphs with a single cycle.

Although this paper is semitutorial, it contains a number of things which have not appeared previously. Beside the generality of our exposition, these include:

- A de-emphasis of a priori graphical models, and an emphasis on algorithms to construct graphical models to fit the given problem.

- A number of nonprobabilistic applications, including the FFT.

- A careful discussion of message scheduling, and a proof of the correctness of a large class of possible schedules.

- A precise measure of the computational complexity of the GDL.

Finally, we note that while this paper was being written, Kschischang, Frey, and Loeliger [41] were simultaneously and independently working out a similar synthesis. And while the final forms of the two papers have turned out to be quite different, anyone interested in the results of this paper should have a look at the alternative formulation in [41].

\section{THE MPF PROBLEM}

The GDL can greatly reduce the number of additions and multiplications required in a certain class of computational problems. It turns out that much of the power of the GDL is due to the fact that it applies to situations in which the notions of addition and multiplication are themselves generalized. The appropriate framework for this generalization is the commutative semiring.

Definition: A commutative semiring is a set $K$, together with two binary operations called "+" and ".", which satisfy the following three axioms:

S1. The operation "+" is associative and commutative, and there is an additive identity element called " 0 " such that $k+0=k$ for all $k \in K$. (This axiom makes $(K,+)$ a commutative monoid.)

S2. The operation "." is also associative and commutative, and there is a multiplicative identity element called " 1 " such that $k \cdot 1=k$ for all $k \in K$. (Thus $(K, \cdot)$ is also a commutative monoid.)

S3. The distributive law holds, i.e.,

$$
(a \cdot b)+(a \cdot c)=a \cdot(b+c)
$$

for all triples $(a, b, c)$ from $K$.

The difference between a semiring and a ring is that in a semiring, additive inverses need not exist, i.e., $(K,+)$ is only required to be a monoid, not a group. Thus every commutative ring is automatically a commutative semiring. For example, the set of real or complex numbers, with ordinary addition and multiplication, forms a commutative semiring. Similarly, the set of polynomials in one or more indeterminates over any commutative ring forms a commutative semiring. However, there are many other commutative semirings, some of which are summarized in Table I. (In semirings 4-8, the set $K$ is an interval of real numbers with the possible addition of $\pm \infty$.) 
TABLE I

Some Commutative Semirings. Here $A$ DENOTES AN ARBITRARY COMMUTATIVE RING, $S$ IS AN ARBITRARY FinITE Set, AND $\Lambda$ Denotes an ARbitrary Distributive LatTice

\begin{tabular}{|c|c|c|c|c|}
\hline & $K$ & $"(+, 0) "$ & $"(\cdot, 1) "$ & short name \\
\hline 1. & $A$ & $(+, 0)$ & $(\cdot, 1)$ & \\
\hline 2. & $A[x]$ & $(+, 0)$ & $(\cdot, 1)$ & \\
\hline 3. & $A[x, y, \ldots]$ & $(+, 0)$ & $(\cdot, 1)$ & \\
\hline 4. & {$[0, \infty)$} & $(+, 0)$ & $(\cdot, 1)$ & sum-product \\
\hline 5. & $(0, \infty]$ & $(\min , \infty)$ & $(\cdot, 1)$ & min-product \\
\hline 6. & {$[0, \infty)$} & $(\max , 0)$ & $(\cdot, 1)$ & max-product \\
\hline 7. & $(-\infty, \infty]$ & $(\min , \infty)$ & $(+, 0)$ & min-sum \\
\hline 8. & {$[-\infty, \infty)$} & $(\max ,-\infty)$ & $(+, 0)$ & max-sum \\
\hline 9. & $\{0,1\}$ & $(\mathrm{OR}, 0)$ & (AND, 1) & Boolean \\
\hline 10. & $2^{S}$ & $(\cup, \emptyset)$ & $(\cap, S)$ & \\
\hline 11. & $\Lambda$ & $(\vee, 0)$ & $(\wedge, 1)$ & \\
\hline 12. & $\Lambda$ & $(\wedge, 1)$ & $(v, 0)$ & \\
\hline
\end{tabular}

For example, consider the min-sum semiring in Table I (entry 7). Here $K$ is the set of real numbers, plus the special symbol " $\infty$." The operation " + " is defined as the operation of taking the minimum, with the symbol $\infty$ playing the role of the corresponding identity element, i.e., we define $\min (k, \infty)=k$ for all $k \in K$. The operation "." is defined to be ordinary addition [sic], with the real number 0 playing the role of the identity, and $k+\infty=\infty$ for all $k$. Oddly enough, this combination forms a semiring, because the distributive law is equivalent to

$$
\min (a+b, a+c)=a+\min (b, c)
$$

which is easily seen to be true. We shall get a glimpse of the importance of this semiring in Examples 2.3 and 4.3, below. (In fact, semirings 5-8 are all isomorphic to each other; for example, 5 becomes 6 via the mapping $x \rightarrow 1 / x$, and 6 becomes 7 under the mapping $x \rightarrow-\log x$.)

Having briefly discussed commutative semirings, we now describe the "marginalize a product function" problem, which is a general computational problem solved by the GDL. At the end of the section we will give several examples of the MPF problem, which demonstrate how it can occur in a surprisingly wide variety of settings.

Let $x_{1}, \cdots, x_{n}$ be variables taking values in the finite sets $A_{1}, \cdots, A_{n}$, with $\left|A_{i}\right|=q_{i}$ for $i=1, \cdots, n$. If $S=\left\{i_{1}, \cdots, i_{r}\right\}$ is a subset of $\{1, \cdots, n\}$, we denote the product $A_{i_{1}} \times \cdots \times A_{i_{r}}$ by $A_{S}$, the variable list $\left(x_{i_{1}}, \cdots, x_{i_{r}}\right)$ by $x_{S}$, and the cardinality of $A_{S}$, i.e., $\left|A_{S}\right|$, by $q_{S}$. We denote the product $A_{\{1, \cdots, n\}}$ simply by $\boldsymbol{A}$, and the variable list $\left\{x_{1}, \cdots, x_{n}\right\}$ simply by $\boldsymbol{x}$.

Now let $\mathcal{S}=\left\{S_{1}, \cdots, S_{M}\right\}$ be $M$ subsets of $\{1, \cdots, n\}$. Suppose that for each $i=1, \cdots, M$, there is a function $\alpha_{i}: A_{S_{i}} \rightarrow R$, where $R$ is a commutative semiring. The variable lists $x_{S_{i}}$ are called the local domains and the functions $\alpha_{i}$ are called the local kernels. We define the global kernel $\beta: A \rightarrow R$ as follows:

$$
\beta\left(x_{1}, \cdots, x_{n}\right)=\prod_{i=1}^{M} \alpha_{i}\left(x_{S_{i}}\right) .
$$

With this setup, the MPF problem is this: For one or more of the indices $i=1, \cdots, M$, compute a table of the values of the
$S_{i}$-marginalization of the global kernel $\beta$, which is the function $\beta_{i}: A_{S_{i}} \rightarrow R$, defined by

$$
\beta_{i}\left(x_{S_{i}}\right)=\sum_{x_{S_{i}^{c}} \in A_{S_{i}^{c}}} \beta(\boldsymbol{x}) .
$$

In (2.2), $S_{i}^{c}$ denotes the complement of the set $S_{i}$ relative to the "universe" $\{1, \cdots, n\}$. For example, if $n=4$, and if $S_{i}=$ $\{1,4\}$, then

$$
\beta_{i}\left(x_{1}, x_{4}\right)=\sum_{x_{2} \in A_{2}, x_{3} \in A_{3}} \beta\left(x_{1}, x_{2}, x_{3}, x_{4}\right) .
$$

We will call the function $\beta_{i}\left(x_{S_{i}}\right)$ defined in (2.2) the $i$ th objective function, or the objective function at $S_{i}$. We note that the computation of the $i$ th objective function in the obvious way requires $q_{1} q_{2} \cdots q_{n}$ additions and $(M-1) q_{1} q_{2} \cdots q_{n}$ multiplications, for a total of $M q_{1} q_{2} \cdots q_{n}$ arithmetic operations, where $q_{i}$ denotes the size of the set $A_{i}$. We shall see below (Section V) that the algorithm we call the "generalized distributive law" can often reduce this figure dramatically.

We conclude this section with some illustrative examples of the MPF problem.

Example 2.1: Let $x_{1}, x_{2}, x_{3}$, and $x_{4}$ be variables taking values in the finite sets $A_{1}, A_{2}, A_{3}$, and $A_{4}$. Suppose $f\left(x_{1}, x_{2}, x_{4}\right)$ and $g\left(x_{1}, x_{3}\right)$ are given functions of these variables, and that it is desired to compute tables of the functions $\alpha\left(x_{1}, x_{4}\right)$ and $\beta\left(x_{2}\right)$ defined by

$$
\begin{aligned}
\alpha\left(x_{1}, x_{4}\right) & =\sum_{x_{2} \in A_{2}, x_{3} \in A_{3}} f\left(x_{1}, x_{2}, x_{4}\right) g\left(x_{1}, x_{3}\right) \\
\beta\left(x_{2}\right) & =\sum_{x_{1} \in A_{1}, x_{3} \in A_{3}, x_{4} \in A_{4}} f\left(x_{1}, x_{2}, x_{4}\right) g\left(x_{1}, x_{3}\right) .
\end{aligned}
$$

ptThis is an instance of the MPF problem, if we define local domains and kernels as follows:

$$
\begin{array}{ccc} 
& \text { local domain } & \text { local kernel } \\
\text { 1. } & \left\{x_{1}, x_{2}, x_{4}\right\} & f\left(x_{1}, x_{2}, x_{4}\right) \\
\text { 2. } & \left\{x_{1}, x_{3}\right\} & g\left(x_{1}, x_{3}\right) \\
\text { 3. } & \left\{x_{1}, x_{4}\right\} & 1 \\
\text { 4. } & \left\{x_{2}\right\} & 1
\end{array}
$$

The desired function $\alpha\left(x_{1}, x_{4}\right)$ is the objective function at local domain 3 , and $\beta\left(x_{2}\right)$ is the objective function at local domain 4. This is just a slightly altered version of Example 1.1, and we shall see in Section IV that when the GDL is applied, the "algorithm" of Example 1.1 results.

Example 2.2: Let $x_{1}, x_{2}, x_{3}, y_{1}, y_{2}$, and $y_{3}$ be six variables, each assuming values in the binary set $\{0,1\}$, and let $f\left(y_{1}, y_{2}, y_{3}\right)$ be a real-valued function of the variables $y_{1}$, $y_{2}$, and $y_{3}$. Now consider the MPF problem (the commutative semiring being the set of real numbers with ordinary addition and multiplication) with the following local domains and kernels:

$\begin{array}{lcc} & \text { local domain } & \text { local kernel } \\ \text { 1. } & \left\{y_{1}, y_{2}, y_{3}\right\} & f\left(y_{1}, y_{2}, y_{3}\right) \\ \text { 2. } & \left\{x_{1}, y_{1}\right\} & (-1)^{x_{1} y_{1}} \\ \text { 3. } & \left\{x_{2}, y_{2}\right\} & (-1)^{x_{2} y_{2}} \\ \text { 4. } & \left\{x_{3}, y_{3}\right\} & (-1)^{x_{3} y_{3}} \\ \text { 5. } & \left\{x_{1}, x_{2}, x_{3}\right\} & 1\end{array}$


Here the global kernel, i.e., the product of the local kernels, is

$$
\begin{aligned}
F\left(x_{1}, x_{2}, x_{3}, y_{1}, y_{2}, y_{3}\right)= & f\left(y_{1}, y_{2}, y_{3}\right) \\
& \cdot(-1)^{x_{1} y_{1}+x_{2} y_{2}+x_{3} y_{3}}
\end{aligned}
$$

and the objective function at the local domain $\left\{x_{1}, x_{2}, x_{3}\right\}$ is

$$
F\left(x_{1}, x_{2}, x_{3}\right)=\sum_{y_{1}, y_{2}, y_{3}} f\left(y_{1}, y_{2}, y_{3}\right)(-1)^{x_{1} y_{1}+x_{2} y_{2}+x_{3} y_{3}}
$$

which is the Hadamard transform of the original function $f\left(y_{1}, y_{2}, y_{3}\right)$ [17]. Thus the problem of computing the Hadamard transform is a special case of the MPF problem. (A straightforward generalization of this example shows that the problem of computing the Fourier transform over any finite Abelian group is also a special case of the MPF problem. While the kernel for the Hadamard transform is of diagonal form, in general, the kernel will only be lower triangular. See [1, Ch. 3] for the details.) We shall see below in Example 4.2 that the GDL algorithm, when applied to this set of local domains and kernels, yields the fast Hadamard transform.

Example 2.3: (Wiberg [39]). Consider the $(7,4,2)$ binary linear code defined by the parity-check matrix

$$
H=\left(\begin{array}{lllllll}
1 & 1 & 0 & 1 & 0 & 0 & 0 \\
0 & 0 & 1 & 1 & 0 & 1 & 0 \\
0 & 0 & 0 & 1 & 1 & 0 & 1
\end{array}\right)
$$

Suppose that an unknown codeword $\left(x_{1}, x_{2}, \cdots, x_{7}\right)$ from this code is transmitted over a discrete memoryless channel, and that the vector $\left(y_{1}, y_{2}, \cdots, y_{7}\right)$ is received. The "likelihood" of a particular codeword $\left(x_{1}, x_{2}, \cdots, x_{7}\right)$ is then

$$
p\left(y_{1}, \cdots, y_{7} \mid x_{1}, \cdots, x_{7}\right)=\prod_{i=1}^{7} p\left(y_{i} \mid x_{i}\right)
$$

where the $p\left(y_{i} \mid x_{i}\right)$ 's are the transition probabilities of the channel. The maximum-likelihood decoding problem is that of finding the codeword that maximizes the expression in (2.3). Now consider the MPF problem with the following domains and kernels, using the min-sum semiring (semiring 7 from Table I). There is one local domain for each codeword coordinate and one for each row of the parity-check matrix.

$$
\begin{array}{rcc} 
& \text { local domain } & \text { local kernel } \\
\text { 1. } & \left\{x_{1}\right\} & -\log p\left(y_{1} \mid x_{1}\right) \\
\vdots & \vdots & \\
\text { 7. } & \left\{x_{7}\right\} & -\log p\left(y_{7} \mid x_{7}\right) \cdot \\
8 . & \left\{x_{1}, x_{2}, x_{4}\right\} & \chi\left(x_{1}, x_{2}, x_{4}\right) \\
\text { 9. } & \left\{x_{3}, x_{4}, x_{6}\right\} & \chi\left(x_{3}, x_{4}, x_{6}\right) \\
10 . & \left\{x_{4}, x_{5}, x_{7}\right\} & \chi\left(x_{4}, x_{5}, x_{7}\right)
\end{array}
$$

Here $\chi$ is a function that indicates whether a given parity check is satisfied, or not. For example, at the local domain $\left\{x_{1}, x_{2}, x_{4}\right\}$, which corresponds to the first row of the parity-check matrix, we have

$$
\chi\left(x_{1}, x_{2}, x_{4}\right)= \begin{cases}0, & \text { if } x_{1}+x_{2}+x_{4}=0 \\ \infty, & \text { if } x_{1}+x_{2}+x_{4}=1 .\end{cases}
$$

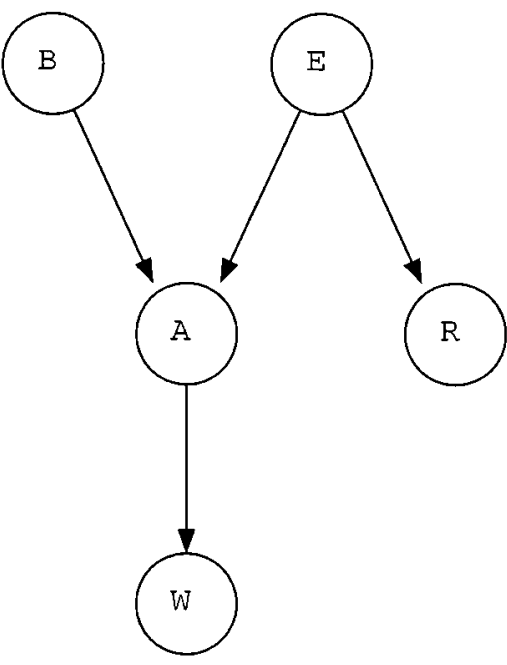

Fig. 1. The Bayesian network in Example 2.4.

The global kernel is then

$$
\begin{aligned}
& F\left(x_{1}, \cdots, x_{7}\right) \\
& \quad=\left\{\begin{array}{cc}
-\log p\left(y_{1}, \cdots, y_{7}\right. & \text { if }\left(x_{1}, \cdots, x_{7}\right) \\
\left.\mid x_{1}, \cdots, x_{7}\right), & \text { is a codeword } \\
\infty, & \text { if }\left(x_{1}, \cdots, x_{7}\right) \\
& \text { is not a codeword. }
\end{array}\right.
\end{aligned}
$$

Thus the objective function at the local domain $\left\{x_{i}\right\}$ is

$$
\begin{array}{r}
F_{i}\left(a_{i}\right)=\min \left\{-\log p\left(y_{1}, \cdots, y_{7} \mid x_{1}, \cdots, x_{7}\right):\right. \\
\text { all codewords for which } \left.x_{i}=a\right\} .
\end{array}
$$

It follows that the value of $a_{i}$ for which $F_{i}\left(a_{i}\right)$ is smallest is the value of the $i$ th component of a maximum-likelihood codeword, i.e., a codeword for which $p\left(y_{1}, \cdots, y_{7} \mid x_{1}, \cdots, x_{7}\right)$ is largest. A straightforward extension of this example shows that the problem of maximum-likelihood decoding of an arbitrary linear block code is a special case of the MPF problem. We shall see in Example 4.3 that when the GDL is applied to problems of this type, the Gallager-Tanner-Wiberg decoding algorithm results.

Example 2.4: Consider the directed acylic graph (DAG) $G$ in Fig. 1. ${ }^{1}$ In a DAG, the "parents" of a vertex $v$, denoted pa $(v)$, are those vertices (if any) which lie immediately "above" $v$. Thus in Fig. 1, pa $(A)=\{B, E\}$, and pa $(B)=\emptyset$. Let us associate a random variable with each of the vertices, and assume that each random variable is dependent only on its "parents," i.e., the joint density function factors as follows:

$$
\begin{aligned}
& \operatorname{Pr}\{B=b, E=e, A=a, R=r, W=w\} \\
& =\operatorname{Pr}\{B=b\} \operatorname{Pr}\{E=e\} \operatorname{Pr}\{A=a \mid B=b, E=e\} \\
& \quad \cdot \operatorname{Pr}\{R=r \mid E=e\} \operatorname{Pr}\{W=w \mid A=a\}
\end{aligned}
$$

${ }^{1}$ This example is taken from [29], in which $B$ stands for burglary, $E$ is for earthquake, $A$ is for alarm sound, $R$ is for radio report, and $W$ is for Watson's call. 


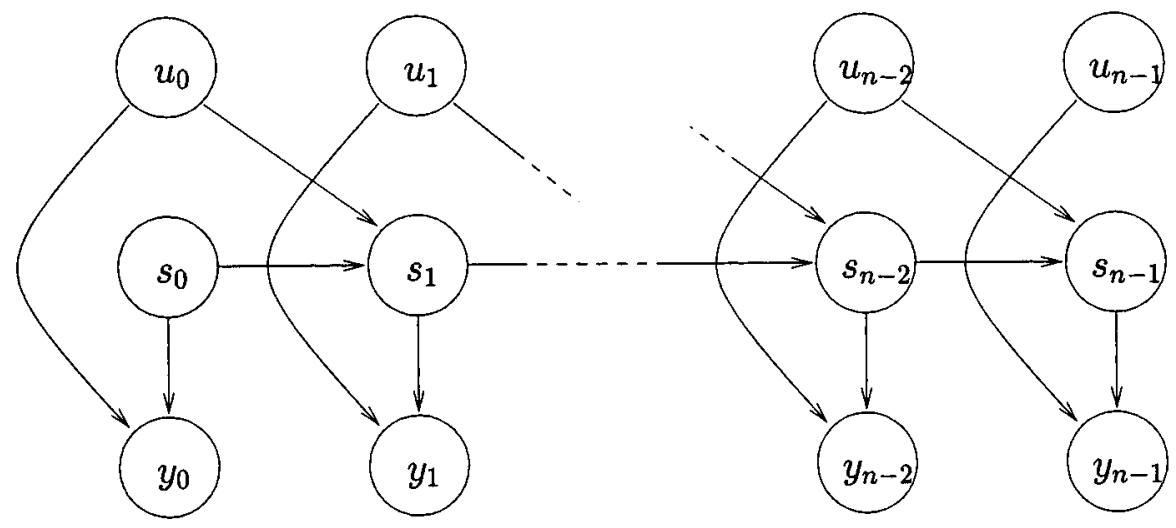

Fig. 2. The Bayesian network for the probabilistic state machine in Example 2.5.

or, using streamlined notation

$$
p(b, e, a, r, w)=p(b) p(e) p(a \mid b, e) p(r \mid e) p(w \mid a) .
$$

A DAG, together with associated random variables whose joint density function factors according to the structure of the DAG, is called a Bayesian network [18].

Let us assume that the two random variables $W$ and $R$ are observed to have the values $w_{0}$ and $r_{0}$, respectively. The probabilistic inference problem, in this case, is to compute the conditional probabilities of one or more of the remaining random variables, i.e., $B, E$, and $A$, where the conditioning is with respect to the "evidence" $\left\{R=r_{0}, W=w_{0}\right\}$. Now consider the MPF problem with the following local domains and kernels:

$$
\begin{array}{ccc} 
& \text { local domain } & \text { local kernel } \\
\text { 1. } & \{b\} & p(b) \\
\text { 2. } & \{e\} & p(e) \\
\text { 3. } & \{a, b, e\} & p(a \mid b, e) \\
\text { 4. } & \{a\} & p\left(w_{0} \mid a\right) \\
\text { 5. } & \{e\} & p\left(r_{0} \mid e\right)
\end{array}
$$

Then by (2.4) (using semiring 4 from Table I, viz. the set of nonnegative real numbers with ordinary addition and multiplication) the global kernel $F(b, e, a)$ is just the function $p\left(b, e, a, r_{0}, w_{0}\right)$, so that, for example, the objective function at local domain 1 is

$$
\begin{aligned}
F_{1}(b) & =\sum_{e, a} p\left(b, e, a, r_{0}, w_{0}\right) \\
& =p\left(b, r_{0}, w_{0}\right) .
\end{aligned}
$$

But by Bayes' rule

$$
p\left(b \mid r_{0}, w_{0}\right)=p\left(b, r_{0}, w_{0}\right) / p\left(w_{0}, r_{0}\right)
$$

so that the conditional probability of $B$, given the "evidence" $\left(r_{0}, w_{0}\right)$, is

$$
\operatorname{Pr}\left\{B=b \mid R=r_{0}, W=w_{0}\right\}=p\left(b \mid r_{0}, w_{0}\right)=\alpha F_{1}(b)
$$

where the constant of proportionality $\alpha$ is given by

$$
\alpha=\left(\sum_{b} F_{1}(b)\right)^{-1} .
$$

Similarly, the computation of the conditional probabilities of $A$ and $E$ can be accomplished via evaluation of the objective functions at the local domains 4 and 5 , respectively. Thus the problem of probabilistic inference in Bayesian networks is a special case of the MPF problem. We shall see in Section IV that when the GDL is applied to problems of this type, the result is an algorithm equivalent to the "probability propagation" algorithms known in the artificial intelligence community.

Example 2.5: As a more useful instance of the probabilistic inference problem, we consider a probabilistic state machine. ${ }^{2}$ At each time $t \in\{0,1, \cdots, n-1\}$, the PSM has state $s_{t}$, input $u_{t}$ and output $y_{t}$. The $u_{t}$ are probabilistically generated, independently, with probabilities $p\left(u_{t}\right)$. The output $y_{t}$ depends on the state $s_{t}$ and input $u_{t}$ and is described by the conditional probability distribution $p\left(y_{t} \mid s_{t}, u_{t}\right)$. The state $s_{t+1}$ also depends on $s_{t}$ and $u_{t}$, with conditional probability $p\left(s_{t+1} \mid s_{t}, u_{t}\right)$. If the $a$ priori distribution of the initial state $s_{0}$ is known, we can write the joint probability of the inputs, states, and outputs from time 0 to $n-1$ as

$$
\begin{aligned}
p\left(u_{0}, \cdots, u_{n-1}, s_{0}, \cdots, s_{n-1}, y_{0}, \cdots, y_{n-1}\right) & =p\left(s_{0}\right) p\left(u_{0}\right) p\left(y_{0} \mid s_{0}, u_{0}\right) \\
& \cdot \prod_{t=1}^{n-1} p\left(s_{t} \mid s_{t-1}, u_{t-1}\right) p\left(u_{t}\right) p\left(y_{t} \mid s_{t}, u_{t}\right) .
\end{aligned}
$$

This means that the PSM is a Bayesian network, as depicted in Fig. 2.

Suppose we observe the $n$ output values, denoting these observations by $y_{0}^{e}, \cdots, y_{n-1}^{e}$ (" $e$ " for evidence), and wish to infer the values of the inputs based on this evidence. This is a probabilistic inference problem of the type discussed in Example 2.4. We can compute the conditional probability

\footnotetext{
${ }^{2}$ Our probabilistic state machines are closely related to the "hidden Markov models" considered in the literature [32].
} 
$\operatorname{Pr}\left\{u_{t}=a \mid y_{0}^{e}, \cdots, y_{n-1}^{e}\right\}$ by taking the joint probability in (2.5) with the observed values of $y_{t}^{e}$ and marginalizing out all the $s_{t}$ 's and all but one of the $u_{t}$ 's. This is an instance of the MPF problem, with the following local domains and kernels (illustrated for $n=4$ ):

$\begin{array}{rcc} & \text { local domain } & \text { local kernel } \\ \text { 1. } & \left\{u_{0}\right\} & p\left(u_{0}\right) \\ \text { 2. } & \left\{u_{1}\right\} & p\left(u_{1}\right) \\ \text { 3. } & \left\{u_{2}\right\} & p\left(u_{2}\right) \\ \text { 4. } & \left\{u_{3}\right\} & p\left(u_{3}\right) \\ \text { 5. } & \left\{s_{0}\right\} & p\left(s_{0}\right) \\ 6 . & \left\{u_{0}, s_{0}\right\} & p\left(y_{0}^{e} \mid u_{0}, s_{0}\right) \\ \text { 7. } & \left\{u_{1}, s_{1}\right\} & p\left(y_{1}^{e} \mid u_{1}, s_{1}\right) \\ 8 . & \left\{u_{2}, s_{2}\right\} & p\left(y_{2}^{e} \mid u_{2}, s_{2}\right) \\ 9 . & \left\{u_{3}, s_{3}\right\} & p\left(y_{3}^{e} \mid u_{3}, s_{3}\right) \\ \text { 10. } & \left\{u_{0}, s_{0}, s_{1}\right\} & p\left(s_{1} \mid u_{0}, s_{0}\right) \\ \text { 11. } & \left\{u_{1}, s_{1}, s_{2}\right\} & p\left(s_{2} \mid u_{1}, s_{1}\right) \\ \text { 12. } & \left\{u_{2}, s_{2}, s_{3}\right\} & p\left(s_{3} \mid u_{2}, s_{2}\right)\end{array}$

This model includes, as a special case, convolutional codes, as follows. The state transition is deterministic, which means that $p\left(s_{t+1} \mid s_{t}, u_{t}\right)=1$ when $s_{t+1}=s_{t+1}\left(s_{t}, u_{t}\right)$ and $p\left(s_{t+1} \mid s_{t}, u_{t}\right)=0$ otherwise. Assuming a memoryless channel, the output $y_{t}$ is probabilistically dependent on $x_{t}$, which is a deterministic function of the state and input, and so $p\left(y_{t} \mid s_{t}, u_{t}\right)=p\left(y_{t} \mid x_{t}\left(s_{t}, u_{t}\right)\right)$. Marginalizing the product of functions in (2.5) in the sum-product and max-product semirings will then give us the maximum-likelihood input symbols or input block, respectively. As we shall see below (Example 4.5), when the GDL is applied here, we get algorithms equivalent to the BCJR and Viterbi decoding algorithms. ${ }^{3}$

Example 2.6: Let $M_{i}$ be a $q_{i-1} \times q_{i}$ matrix with entries in a commutative semiring, for $i=1, \cdots, n$. We denote the entries in $M_{i}$ by $M_{i}\left[x_{i-1}, x_{i}\right]$, where for $i=0,1, \cdots, n, x_{i}$ is a variable taking values in a set $A_{i}$ with $q_{i}$ elements. Suppose we want to compute the product

$$
M=M_{1} \cdot M_{2} \cdots M_{n} .
$$

Then for $n=2$ we have by definition

$$
M\left[x_{0}, x_{2}\right]=\sum_{x_{1}} M_{1}\left[x_{0}, x_{1}\right] M_{2}\left[x_{1}, x_{2}\right]
$$

and an easy induction argument gives the generalization

$$
M\left[x_{0}, x_{n}\right]=\sum_{x_{1}, \cdots, x_{n-1}} M_{1}\left[x_{0}, x_{1}\right] \cdots M_{n}\left[x_{n-1}, x_{n}\right] .
$$

(Note that (2.7) suggests that the number of arithmetic operations required to multiply these $n$ matrices is $2 q_{0} q_{1} \cdots q_{n}$.) Thus

\footnotetext{
${ }^{3}$ To obtain an algorithm equivalent to Viterbi's, it is necessary to take the negative logarithm of (2.5) before performing the marginalization in the min-sum semiring.
}

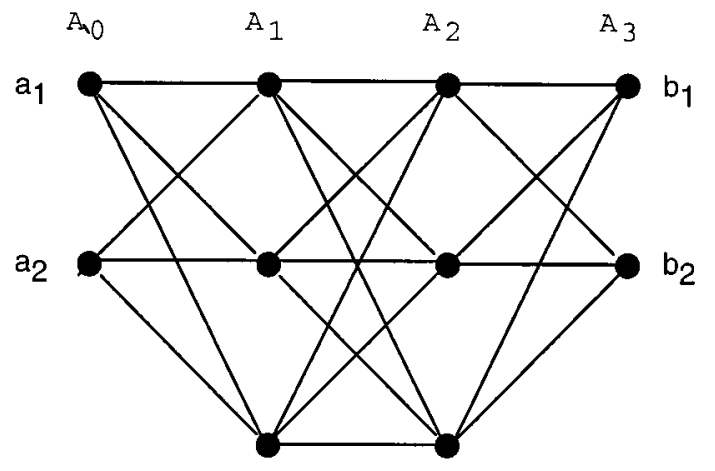

Fig. 3. The trellis corresponding to the multiplication of three matrices, of sizes $2 \times 3,3 \times 3$, and $3 \times 2$. The $(i, j)$ th entry in the matrix product is the sum of the weights of all paths from $a_{i}$ to $b_{j}$.

multiplying $n$ matrices can be formulated as the following MPF problem:

$$
\begin{array}{ccc} 
& \text { local domain } & \text { local kernel } \\
\text { 1. } & \left\{x_{0}, x_{1}\right\} & M_{1}\left[x_{0}, x_{1}\right] \\
2 . & \left\{x_{1}, x_{2}\right\} & M_{2}\left[x_{1}, x_{2}\right] \\
& \vdots & \\
& \vdots & \\
n & \left\{x_{n-1}, x_{n}\right\} & M_{n}\left[x_{n-1}, x_{n}\right] \\
n+1 & \left\{x_{0}, x_{n}\right\} & 1
\end{array}
$$

the desired result being the objective function at local domain $n+1$.

As an alternative interpretation of (2.7), consider a trellis of depth $n$, with vertex set $A_{0} \cup A_{1} \cdots \cup A_{n}$, and an edge of weight $M_{i}\left[x_{i-1}, x_{i}\right]$ connecting the vertices $x_{i-1} \in A_{i-1}$ and $x_{i} \in A_{i}$. If we define the weight of a path as the sum of the weights of the component edges, then $M\left[x_{0}, x_{n}\right]$ as defined in (2.7) represents the sum of the weights of all paths from $x_{0}$ to $x_{n}$. For example, Fig. 3 shows the trellis corresponding to the multiplication of three matrices, of sizes $2 \times 3,3 \times 3$, and $3 \times 2$. If the computation is done in the min-sum semiring, the interpretation is that $M\left[x_{0}, x_{n}\right]$ is the weight of a minimum-weight path from $x_{0}$ to $x_{n}$.

We shall see in Section IV that if the GDL is applied to the matrix multiplication problem, a number of different algorithms result, corresponding to the different ways of parenthesizing the expression $M_{1} M_{2} \cdots M_{n}$. If the parenthesization is

$$
\left(\left(\left(M_{1} M_{2}\right) M_{3}\right) M_{4}\right) M_{5}
$$

(illustrated for $n=5$ ), and the computation is in the min-sum semiring, Viterbi's algorithm results.

\section{The GDL: An Algorithm FOR Solving THE MPF PROBLEM}

If the elements of $\mathcal{S}$ stand in a certain special relationship to each other, then an algorithm for solving the MPF problem can be based on the notion of "message passing." The required relationship is that the local domains can be organized into a junction tree [18]. What this means is that the elements of $\mathcal{S}$ 
4

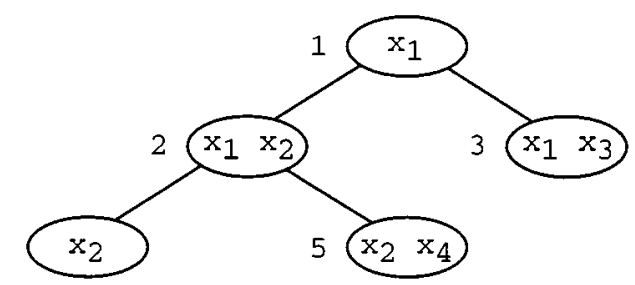

Fig. 4. A junction tree.

can be attached as labels to the vertices of a graph-theoretic tree $T$, such that for any two vertices $v_{i}$ and $v_{j}$, the intersection of the corresponding labels, viz. $S_{i} \cap S_{j}$, is a subset of the label on each vertex on the unique path from $v_{i}$ to $v_{j}$. Alternatively, the subgraph of $T$ consisting of those vertices whose label includes the element $i$, together with the edges connecting these vertices, is connected, for $i=1, \cdots, n$.

For example, consider the following five local domains:

$\begin{array}{lc} & \text { local domain } \\ \text { 1. } & \left\{x_{1}\right\} \\ \text { 2. } & \left\{x_{1}, x_{2}\right\} \\ \text { 3. } & \left\{x_{1}, x_{3}\right\} \\ \text { 4. } & \left\{x_{2}\right\} \\ \text { 5. } & \left\{x_{2}, x_{4}\right\}\end{array}$

These local domains can be organized into a junction tree, as shown in Fig. 4. For example, the unique path from vertex 2 to vertex 3 is $v_{2} \rightarrow v_{1} \rightarrow v_{3}$, and $S_{2} \cap S_{3} \subseteq S_{1}$, as required.

On the other hand, the following set of four local domains cannot be organized into a junction tree, as can be easily verified.

$$
\begin{array}{lc} 
& \text { local domain } \\
\text { 1. } & \left\{x_{1}, x_{2}\right\} \\
\text { 2. } & \left\{x_{2}, x_{3}\right\} \\
\text { 3. } & \left\{x_{3}, x_{4}\right\} \\
\text { 4. } & \left\{x_{1}, x_{4}\right\}
\end{array}
$$

However, by adjoining two "dummy domains"

$$
\begin{array}{ll} 
& \text { local domain } \\
\text { 5. } & \left\{x_{1}, x_{2}, x_{4}\right\} \\
\text { 6. } & \left\{x_{2}, x_{3}, x_{4}\right\}
\end{array}
$$

to the collection, we can devise a junction tree, as shown in Fig. 5 .

(In Section IV, we give a simple algorithm for deciding whether or not a given set of local domains can be organized into a junction tree, for constructing one if it does exist, and for finding appropriate dummy domains if it does not.)

In the "junction tree" algorithm, which is what we call the generalized distributive law (GDL), if $v_{i}$ and $v_{j}$ are connected by an edge (indicated by the notation $v_{i}$ adj $v_{j}$ ), the (directed) "message" from $v_{i}$ to $v_{j}$ is a table containing the values of a function $\mu_{i, j}: A_{S_{i} \cap S_{j}} \rightarrow R$. Initially, all such functions are defined to be identically 1 (the semiring's multiplicative identity);

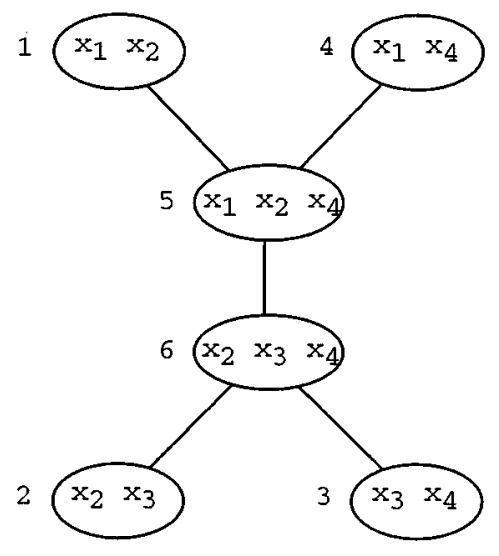

Fig. 5. A junction tree which includes the local domains $\left\{x_{1}, x_{2}\right\},\left\{x_{2}, x_{3}\right\}$, $\left\{x_{3}, x_{4}\right\}$, and $\left\{x_{1}, x_{4}\right\}$.

and when a particular message $\mu_{i, j}$ is updated, the following rule is used:

$\mu_{i, j}\left(x_{S_{i} \cap S_{j}}\right)=\sum_{x_{S_{i} \backslash S_{j}} \in A_{S_{i} \backslash S_{j}}} \alpha_{i}\left(x_{S_{i}}\right) \prod_{\substack{v_{k} \\ k \neq j j}} \mu_{k, i}\left(x_{S_{k} \cap S_{i}}\right)$.

A good way to remember (3.1) is to think of the junction tree as a communication network, in which an edge from $v_{i}$ to $v_{j}$ is a transmission line that "filters out" dependence on all variables but those common to $v_{i}$ and $v_{j}$. (The filtering is done by marginalization.) When the vertex $v_{i}$ wishes to send a message to $v_{j}$, it forms the product of its local kernel with all messages it has received from its neighbors other than $v_{j}$, and transmits the product to $v_{j}$ over the $\left(v_{i}, v_{j}\right)$ transmission line.

Similarly, the "state" of a vertex $v_{i}$ is defined to be a table containing the values of a function $\sigma_{i}: A_{S_{i}} \rightarrow R$. Initially, $\sigma_{i}$ is defined to be the local kernel $\alpha_{i}\left(x_{S_{i}}\right)$, but when $\sigma_{i}$ is updated, the following rule is used:

$$
\sigma_{i}\left(x_{S_{i}}\right)=\alpha_{i}\left(x_{S_{i}}\right) \prod_{v_{k} \text { adj } v_{i}} \mu_{k, i}\left(x_{S_{k} \cap S_{i}}\right) .
$$

In words, the state of a vertex $v_{i}$ is the product of its local kernel with each of the messages it has received from its neighbors. The basic idea is that after sufficiently many messages have been passed, $\sigma_{i}\left(x_{S_{i}}\right)$ will be the objective function at $S_{i}$, as defined in (2.2).

The question remains as to the scheduling of the message passing and the state computation. Here we consider only two special cases, the single-vertex problem, in which the goal is to compute the objective function at only one vertex $v_{0}$, and the all-vertices problem, where the goal is to compute the objective function at all vertices. ${ }^{4}$

For the single-vertex problem, the natural (serial) scheduling of the GDL begins by directing each edge toward the

\footnotetext{
${ }^{4}$ We do not consider the problem of evaluating the objective function at $k$ vertices, where $1<k<M$. However, as we will see in Section $\mathrm{V}$, the complexity of the $M$-vertex GDL is at most four times as large as the 1 -vertex GDL, so it is reasonably efficient to solve the $k$-vertex problem using the $M$-vertex solution.
} 


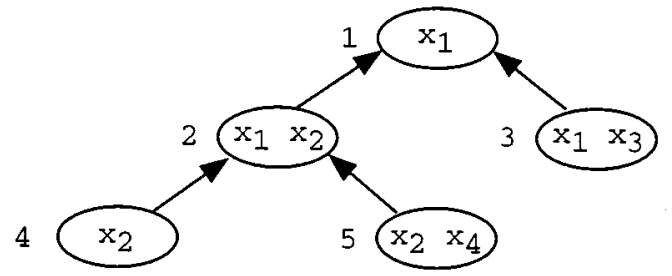

Fig. 6. The junction tree of Fig. 4 with the edges directed towards $v_{1}$.

TABLE II

A SChEdUle For the SingLE-VERTEX GDL FOR THE Junction TREe OF Fig. 4, WITH TARGET VERTEX $v_{1}$

Round Message or State Computation

$\begin{array}{ll}1 & \mu_{3,1}\left(x_{1}\right)=\sum_{x_{3}} \alpha_{3}\left(x_{1}, x_{3}\right) \\ 2 & \mu_{4,2}\left(x_{2}\right)=\alpha_{4}\left(x_{2}\right) \\ 3 & \mu_{5,2}\left(x_{2}\right)=\sum_{x_{4}} \alpha_{5}\left(x_{2}, x_{4}\right) \\ 4 & \mu_{2,1}\left(x_{1}\right)=\sum_{x_{2}} \alpha_{2}\left(x_{1}, x_{2}\right) \cdot \mu_{4,2}\left(x_{2}\right) \cdot \mu_{5,2}\left(x_{2}\right) \\ 5 & \sigma_{1}\left(x_{1}\right)=\alpha_{1}\left(x_{1}\right) \cdot \mu_{2,1}\left(x_{1}\right) \cdot \mu_{3,1}\left(x_{1}\right) .\end{array}$

target vertex $v_{0}$. Then messages are sent only in the direction toward $v_{0}$, and each directed message is sent only once. A vertex sends a message to a neighbor, when, for the first time, it has received messages from each of its other neighbors. The target $v_{0}$ computes its state when it has received messages from each of its neighbors. With this scheduling, messages begin at the leaves (vertices with degree 1), and proceed toward $v_{0}$, until $v_{0}$ has received messages from all its neighbors, at which point $v_{0}$ computes its state and the algorithm terminates.

For example, if we wish to solve the single-vertex problem for the junction tree of Fig. 4, and the target vertex is $v_{1}$, the edges should all be directed towards $v_{1}$, as shown in Fig. 6. Then one possible sequence of messages and state computations runs as shown in Table II.

It will be shown in Section $\mathrm{V}$ that this scheduling of the single vertex GDL requires at most

$$
\sum_{v} d(v)\left|A_{S(v)}\right| \quad \text { arithmetic operations }
$$

where $S(v)$ is the label of $v$, and $d(v)$, the degree of $v$, is the number of vertices adjacent to $v$. This should be compared to the complexity of the "obvious" solution, which as we noted above is $M q_{1} \cdots q_{n}$ operations. For example, for the junction tree shown in Fig. 6, the complexity of the single-vertex GDL is by (3.3) at most $2 q_{1}+3 q_{1} q_{2}+q_{1} q_{3}+q_{2}+q_{2} q_{4}$ arithmetic operations, versus $5 q_{1} q_{2} q_{3} q_{4} q_{4}$ for the direct computation.

For the all-vertices problem, the GDL can be scheduled in several ways. For example, in a fully parallel implementation, at every iteration, every state is updated, and every message is computed and transmitted, simultaneously. In this case the messages and states will stabilize after a number of iterations
TABLE III

A "HyBRID" SCHEDULE FOR THE ALL-VERTICES GDL FOR THE JUNCTION TREE OF Fig. 4

\begin{tabular}{cl} 
Round & Message and State Computations \\
\hline \multirow{2}{*}{1} & $\mu_{3,1}\left(x_{1}\right)=\sum_{x_{3}} \alpha_{3}\left(x_{1}, x_{3}\right)$ \\
& $\mu_{4,2}\left(x_{2}\right)=\alpha_{4}\left(x_{2}\right)$ \\
& $\mu_{5,2}\left(x_{2}\right)=\sum_{x_{4}} \alpha_{5}\left(x_{2}, x_{4}\right)$ \\
2 & $\mu_{1,2}\left(x_{1}\right)=\sum_{x_{2}} \alpha_{1}\left(x_{1}, x_{2}\right) \cdot \mu_{3,1}\left(x_{1}\right)$ \\
& $\mu_{2,1}\left(x_{1}\right)=\sum_{x_{2}} \alpha_{2}\left(x_{1}, x_{2}\right) \cdot \mu_{4,2}\left(x_{2}\right) \cdot \mu_{5,2}\left(x_{2}\right)$ \\
3 & $\mu_{1,3}\left(x_{1}\right)=\alpha_{1}\left(x_{1}\right) \cdot \mu_{2,1}\left(x_{1}\right)$ \\
& $\mu_{2,4}\left(x_{2}\right)=\sum_{x_{1}} \alpha_{2}\left(x_{1}, x_{2}\right) \cdot \mu_{1,2}\left(x_{1}\right) \cdot \mu_{5,2}\left(x_{2}\right)$ \\
& $\mu_{2,5}\left(x_{2}\right)=\sum_{x_{1}} \alpha_{2}\left(x_{1}, x_{2}\right) \cdot \mu_{1,2}\left(x_{1}\right) \cdot \mu_{4,2}\left(x_{2}\right)$ \\
& $\sigma_{1}\left(x_{1}\right)=\alpha_{1}\left(x_{1}\right) \cdot \mu_{2,1}\left(x_{1}\right) \cdot \mu_{3,1}\left(x_{1}\right)$ \\
& $\sigma_{2}\left(x_{1}, x_{2}\right)=\alpha_{2}\left(x_{1}, x_{2}\right) \cdot \mu_{1,2}\left(x_{1}, x_{2}\right) \cdot \mu_{4,2}\left(x_{2}\right) \cdot \mu_{5,2}\left(x_{2}\right)$ \\
& $\sigma_{3}\left(x_{1}, x_{3}\right)=\alpha_{3}\left(x_{1}, x_{3}\right) \cdot \mu_{1,3}\left(x_{1}\right)$ \\
& $\sigma_{4}\left(x_{2}\right)=\alpha_{4}\left(x_{2}\right) \cdot \mu_{2,4}\left(x_{2}\right)$ \\
& $\sigma_{5}\left(x_{2}, x_{4}\right)=\alpha_{5}\left(x_{2}, x_{4}\right) \cdot \mu_{2,5}\left(x_{2}\right)$.
\end{tabular}

at most equal to the diameter of the tree, at which point the states of the vertices will be equal to the desired objective functions, and the algorithm terminates. Alternatively, the GDL can be scheduled fully serially, in which case each message is sent only once, and each state is computed only once. In this case, a vertex sends a message to a neighbor when, for the first time, it has received messages from all of its other neighbors, and computes its state when, for the first time, it has received messages from all its neighbors. In this serial mode, messages begin at the leaves, and proceed inwards into the tree, until some nodes have received messages from all their neighbors, at which point messages propagate outwards, so that each vertex eventually receives messages from all of its neighbors. ${ }^{5}$ We will see in Section IV that the fully serial all-vertices GDL requires at most $4 \sum_{v \in V} d(v)\left|A_{S(v)}\right|$ arithmetic operations.

There are also a variety of possible hybrid schedules, intermediate between fully parallel and fully serial. For example, Table III shows a hybrid schedule for the junction tree of Fig. 4, in which the computation is organized into four rounds. The computations in each round may be performed in any order, or even simultaneously, but the rounds must be performed sequentially.

That concludes our informal discussion of GDL scheduling. We end this section with what we call the "scheduling theorem" for the GDL.

Thus let $T$ be a junction tree with vertex set $V$ and edge set $E$. In the GDL, messages can be passed in both directions on each edge, so it will be convenient to regard the edge set $E$ as consisting of ordered pairs of vertices. Thus for example for the tree of Fig. 4, we have

$$
\begin{aligned}
E=\{(1,2),(2,1),(1,3),(3,1), & \\
& (2,4),(4,2),(2,5),(5,2)\} .
\end{aligned}
$$

A schedule for the GDL is defined to be a finite sequence of subsets of $E$. A typical schedule will be denoted by

${ }^{5}$ We might therefore call the fully serial all-vertices GDL an "inward-outward" algorithm. 


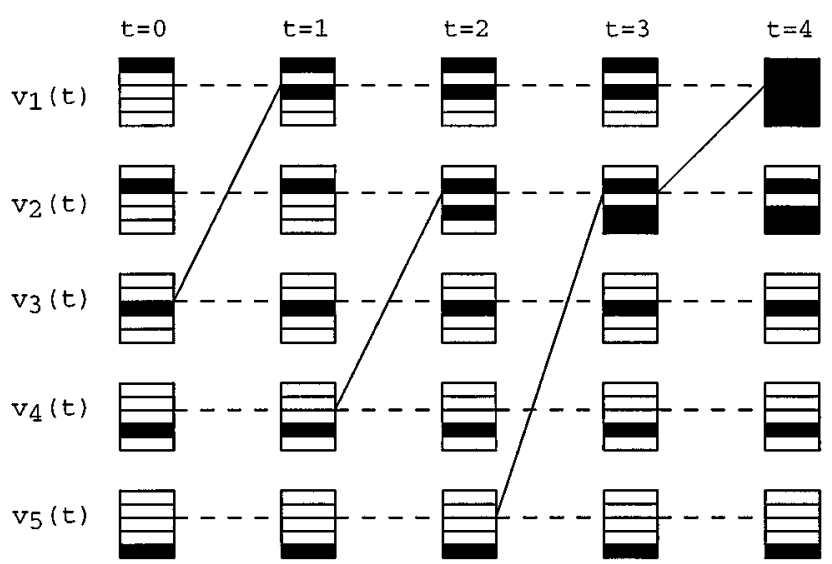

Fig. 7. The message trellis for the junction tree in Fig. 4 under the schedule of Table II viz., $E_{1}=\{(3,1)\}, E_{2}=\{(4,2)\}, E_{3}=\{(5,2)\}, E_{4}=$ $\{(2,1)\}$.

$\mathcal{E}=\left(E_{1}, E_{2}, \cdots, E_{N}\right)$. The idea is that $E_{t}$ is the set of messages that are updated during the $t$ th round of the algorithm. In Tables II and III, for example, the corresponding schedules are

Table II : $\mathcal{E}=(\{(3,1)\},\{(4,2)\},\{(5,2)\},\{(2,1)\})$ Table III : $\mathcal{E}=(\{(3,1),(4,2),(5,2)\},\{(1,2),(2,1)\}$,

$$
\{(1,3),(2,4),(2,5)\}) \text {. }
$$

Given a schedule $\mathcal{E}=\left(E_{1}, E_{2}, \cdots, E_{N}\right)$, the corresponding message trellis is a finite directed graph with vertex set $V \times$ $\{0,1, \cdots, N\}$, in which a typical element is denoted by $v_{i}(t)$, for $t \in\{0,1, \cdots, N\}$. The only allowed edges are of the form $\left(v_{i}(t-1), v_{j}(t)\right)$; and $\left(v_{i}(t-1), v_{j}(t)\right)$ is an edge in the message trellis if either $\left(v_{i}, v_{j}\right) \in E_{t}$ or $i=j$. The message trellises for the junction tree of Fig. 4, under the schedules of Tables II and III, are shown in Figs. 7 and 8, respectively. (In these figures, the shaded boxes indicate which local kernels are known to which vertices at any time. For example, in Fig. 7, we can see that knowledge of the local kernels $\alpha_{2}, \alpha_{4}$, and $\alpha_{5}$ has reached $v_{2}$ at time $t=3$. We will elaborate on this notion of "knowledge" in the Appendix.)

Theorem 3.1 (GDL Scheduling): After the completion of the message passing described by the schedule

$$
\mathcal{E}=\left(E_{1}, E_{2}, \cdots, E_{N}\right)
$$

the state at vertex $v_{j}$ will be the $j$ th objective as defined in (3.2) if and only if there is a path from $v_{i}(0)$ to $v_{j}(N)$ in the corresponding message trellis, for $i=1, \cdots, n$.

A proof of Theorem 3.1 will be found in the Appendix, but Figs. 7 and 8 illustrate the idea. For example, in Fig. 8, we see that there is a path from each of $v_{1}(0), \cdots, v_{5}(0)$ to $v_{2}(2)$, which means (by the scheduling theorem) that after two rounds of message passing, the state at $v_{2}$ will be the desired objective function. This is why, in Table III, we are able to compute $\sigma_{2}$ in round 3. Theorem 3.1 immediately implies the correctness of

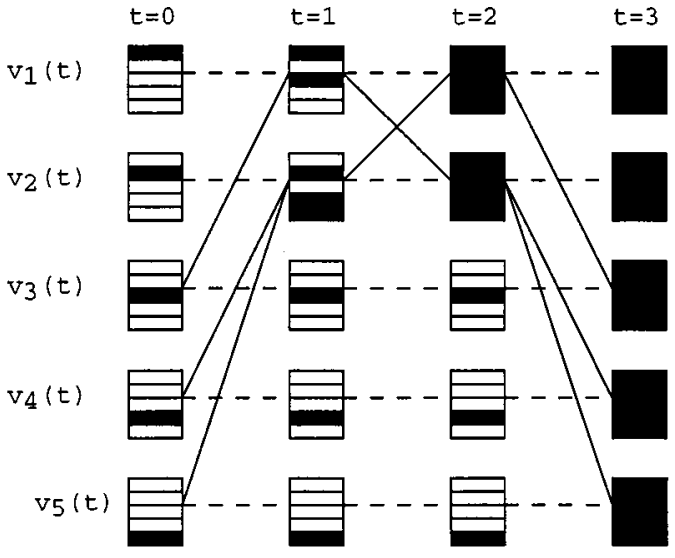

Fig. 8. The message trellis for the junction tree in Fig. 4, under the schedule of Table III, viz., $E_{1}=\{(3,1),(4,2),(5,2)\}, E_{2}=\{(1,2),(2,1)\}$, and $E_{3}=\{(1,3),(2,4),(2,5)\}$.

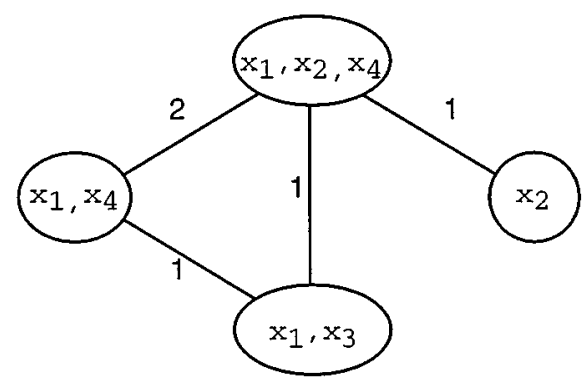

(a)

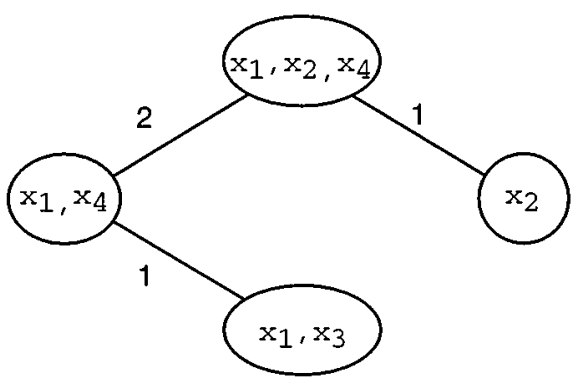

(b)

Fig. 9. (a) The local domain graph and (b) one junction tree for the local domains and kernels in Example 2.1.

the single-vertex and all-vertices serial GDL described earlier in this section.

\section{Constructing Junction Trees}

In Section III we showed that if we can construct a junction tree with the local domains as vertex labels, we can devise a message-passing algorithm to solve the MPF problem. But does such a junction tree exist? And if not, what can be done? In this section we will answer these questions.

It is easy to decide whether or not a junction tree exists. The key is the local domain graph $G_{\mathrm{LD}}$, which is a weighted com- 


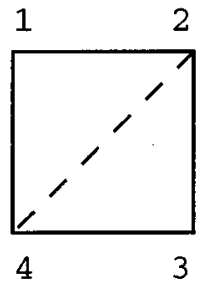

(a)

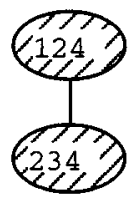

(b)

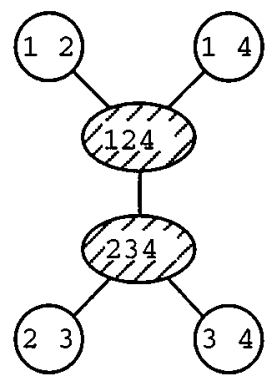

(c)

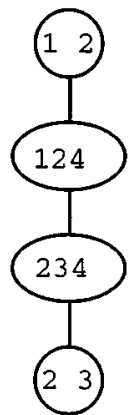

(d)

Fig. 10. Constructing a junction tree for the local domains $\{1,2\},\{2,3\},\{3,4\}$, and $\{4,1\}$ by triangulating the moral graph.

plete graph with $M$ vertices $v_{1}, \cdots, v_{M}$, one for each local domain, with the weight of the edge $e_{i, j}: v_{i} \leftrightarrow v_{j}$ defined by

$$
w_{i, j}=\left|S_{i} \cap S_{j}\right| .
$$

If $x_{k} \in S_{i} \cap S_{j}$, we will say that $x_{k}$ is contained in $e_{i, j}$. Denote by $w_{\max }$ the weight of a maximal-weight spanning tree of $G_{\mathrm{LD}}{ }^{6}$ Finally, define

$$
w^{*}=\sum_{i=1}^{M}\left|S_{i}\right|-n .
$$

Theorem 4.1: $w_{\max } \leq w^{*}$, with equality if and only if there is a junction tree. If $w_{\max }=w^{*}$, then any maximal-weight spanning tree of $G_{\mathrm{LD}}$ is a junction tree.

Proof: For each $k=1, \cdots, n$, denote by $m_{k}$ the number of sets $S_{i}$ which contain the variable $x_{k}$. Note that

$$
\sum_{k=1}^{n} m_{k}=\sum_{i=1}^{M}\left|S_{i}\right| .
$$

Let $T$ be any spanning tree of $G_{\mathrm{LD}}$, and let $w_{k}$ denote the number of edges in $T$ which contain $x_{k}$. Clearly

$$
w(T)=\sum_{k=1}^{n} w_{k} .
$$

Furthermore, $w_{k} \leq m_{k}-1$, since the subgraph $T_{k}$ of $T$ induced by the vertices containing $x_{k}$ has no cycles, and equality holds if and only if $T_{k}$ is connected, i.e., a tree. It follows then that

$$
w(T)=\sum_{k=1}^{n} w_{k} \leq \sum_{k=1}^{n}\left(m_{k}-1\right)=\sum_{i=1}^{M}\left|S_{i}\right|-n=w^{*}
$$

with equality if and only if each subgraph $T_{k}$ is connected, i.e., if $T$ is a junction tree.

Example 4.1: Here we continue Example 2.1. The LD graph is shown in Fig. 9(a). Here $w^{*}=(3+2+2+1)-4=4$. A maximal weight spanning tree is shown in Fig. 9(b), and its weight is 4 , so by Theorem 4.1 , this is a junction tree, a fact

\footnotetext{
${ }^{6}$ A maximal-weight spanning tree can easily be found with Prim's "greedy" algorithm [27, Ch. 3], [9, Sec. 24.2]. In brief, Prim's algorithm works by growing the tree one edge at a time, always choosing a new edge of maximal weight.
}

that can easily be checked directly. If we apply the GDL to this junction tree, we get the "algorithm" described in our introductory Example 1.1 (if we use the schedule $\mathcal{E}=\left\{E_{1}, E_{2}, E_{3}\right\}$, where $E_{1}=\{(4,1),(2,3)\}, E_{2}=\{(1,3),(3,1)\}$, and $E_{3}=$ $\{(1,4)\}$.

If no junction tree exists with the given vertex labels $S_{i}$, all is not lost. We can always find a junction tree with $M$ vertices such that each $S_{i}$ is a subset of the $i$ th vertex label, so that each local kernel $\alpha_{i}$ may be associated with the $i$ th vertex, by regarding it as a function of the variables involved in the label. The key to this construction is the moral graph $^{7}$ which is the undirected graph with vertex set equal to the set of variables $\left\{x_{1}, \cdots, x_{n}\right\}$, and having an edge between $x_{i}$ and $x_{j}$ if there is a local domain which contains both $x_{i}$ and $x_{j}$.

Given a cycle in a graph, a chord is an edge between two vertices on the cycle which do not appear consecutively in the cycle. A graph is triangulated if every simple cycle (i.e., one with no repeated vertices) of length larger than three has a chord.

In [18], it is shown that the cliques (maximal complete subgraphs) of a graph can be the vertex labels of a junction tree if and only if the graph is triangulated. Thus to form a junction tree with vertex labels such that each of the local domains is contained in some vertex label, we form the moral graph, add enough edges to the moral graph so that the resulting graph is triangulated, and then form a junction tree with the cliques of this graph as vertex labels. Each of the original local domains will be a subset of at least one of these cliques. We can then attach the original local domains as "leaves" to the clique junction tree, thereby obtaining a junction tree for the original set of local domains and kernels, plus extra local domains corresponding to the cliques in the moral graph. We can then associate one of the local kernels attached to each of the cliques to that clique, and delete the corresponding leaf. In this way we will have constructed a junction tree for the original set of local kernels, with some of the local domains enlarged to include extra variables. However, this construction is far from unique, and the choices that must be made (which edges to add to the moral graph, how to assign local kernels to the enlarged local domains) make the procedure more of an art than a science.

\footnotetext{
${ }^{7}$ The whimsical term "moral graph" originally referred to the graph obtained from a DAG by drawing edges between--"marrying"- - each of the parents of a given vertex [23].
} 


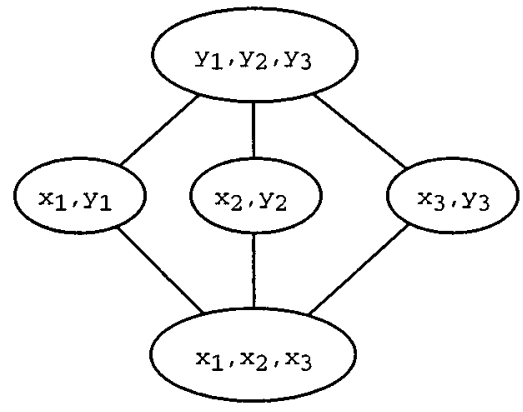

Fig. 11. The LD graph for the local domains and kernels in Example 2.2. (All edges have weight 1.) There is no junction tree.

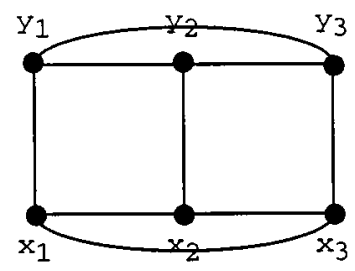

(a)

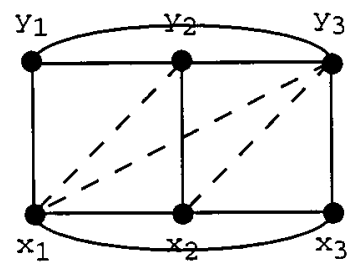

(b)

Fig. 12. The moral graph (top) and a triangulated moral graph (bottom) for the local domains and kernels in Example 2.2.

For example, suppose the local domains and local kernels are

$\begin{array}{ccc} & \text { local domain } & \text { local kernel } \\ \text { 1. } & \left\{x_{1}, x_{2}\right\} & \alpha_{1}\left(x_{1}, x_{2}\right) \\ 2 . & \left\{x_{2}, x_{3}\right\} & \alpha_{2}\left(x_{2}, x_{3}\right) \\ \text { 3. } & \left\{x_{3}, x_{4}\right\} & \alpha_{3}\left(x_{3}, x_{4}\right) \\ \text { 4. } & \left\{x_{1}, x_{4}\right\} & \alpha_{4}\left(x_{1}, x_{4}\right)\end{array}$

As we observed above, these local domains cannot be organized into a junction tree. The moral graph for these domains is shown in Fig. 10(a) (solid lines). This graph is not triangulated, but the addition of the edge 2-4 (dashed line) makes it so. The cliques in the triangulated graph are $\{1,2,4\}$ and $\{2,3,4\}$, and these sets can be made the labels in a junction tree (Fig. 10(b)). We can attach the original four local domains as leaves to this junction tree, as shown in Fig. 10(c) (note that this graph is identical to the junction tree in Fig. 5). Finally, we can assign the local kernel at $\{1,4\}$ to the local domain $\{1,2,4\}$, and the local kernel at $\{3,4\}$ to the local domain $\{2,3,4\}$, thereby obtaining the junction tree shown in Fig. 10(d). What we have done, in effect, is to modify the original local domains by enlarging two

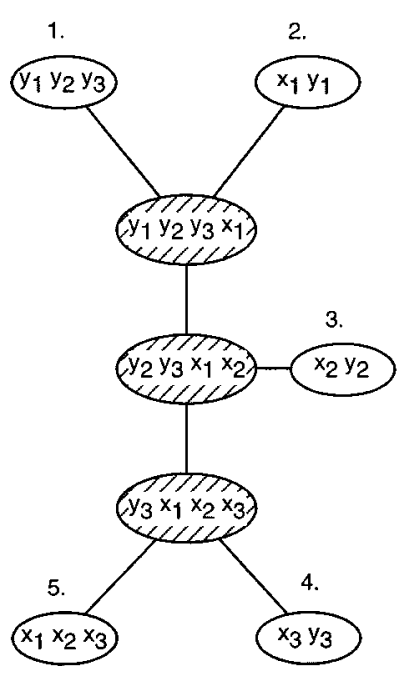

(a)

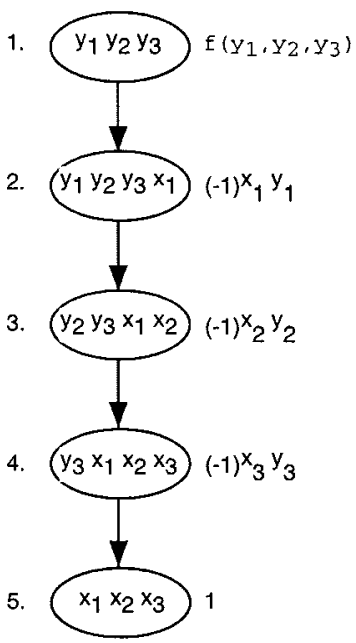

(b)
Fig. 13. Constructing a junction tree for Example 4.2.

of them, and viewing the associated local kernels as functions on the enlarged local domains:

$$
\begin{array}{ccc} 
& \text { local domain } & \text { local kernel } \\
\text { 1. } & \left\{x_{1}, x_{2}\right\} & \alpha_{1}\left(x_{1}, x_{2}\right) \\
\text { 2. } & \left\{x_{2}, x_{3}\right\} & \alpha_{2}\left(x_{2}, x_{3}\right) \\
3^{\prime} . & \left\{x_{2}, x_{3}, x_{4}\right\} & \alpha_{3}^{\prime}\left(x_{2}, x_{3}, x_{4}\right)=\alpha_{3}\left(x_{3}, x_{4}\right) \\
4^{\prime} . & \left\{x_{1}, x_{2}, x_{4}\right\} & \alpha_{4}^{\prime}\left(x_{1}, x_{2}, x_{4}\right)=\alpha_{4}\left(x_{1}, x_{4}\right)
\end{array}
$$

The difficulty is that we must enlarge the local domains enough so that they will support a junction tree, but not so much that the resulting algorithm will be unmanageably complex. We will return to the issue of junction tree complexity in Section V.

The next example illustrates this procedure in a more practical setting.

Example 4.2: Here we continue Example 2.2. The local domain graph is shown in Fig. 11. Since all edges have weight 1, any spanning tree will have weight 4 , but $w^{*}=12-6=6$. Thus by Theorem 4.1, the local domains cannot be organized into a junction tree, so we need to consider the moral graph, which is shown in Fig. 12(a). It is not triangulated (e.g., the cycle formed by vertices $y_{1}, y_{2}, x_{2}$, and $x_{1}$ has no chord), but it can be triangulated by the addition of three additional edges, as shown in Fig. 12(b). There are exactly three cliques in the triangulated moral graph, viz., $\left\{y_{1}, y_{2}, y_{3}, x_{1}\right\},\left\{y_{2}, y_{3}, x_{1}, x_{2}\right\}$, and $\left\{y_{3}, x_{1}, x_{2}, x_{3}\right\}$. These three sets can be organized into a unique junction tree, and each of the original five local domains is a subset of exactly one of these, as shown in Fig. 13(a). If we want a unique local domain for each of the five local kernels, we can retain two of the original local domains, thus obtaining the junction tree shown in Fig. 13(b). Since this is a "single-vertex" problem, to apply the GDL, we first direct each of the edges towards the target vertex, which in this case is $\left\{x_{1}, x_{2}, x_{3}\right\}$. It is now a straightforward exercise to show that the (serial, one-vertex) GDL, when applied to this directed junction tree, 


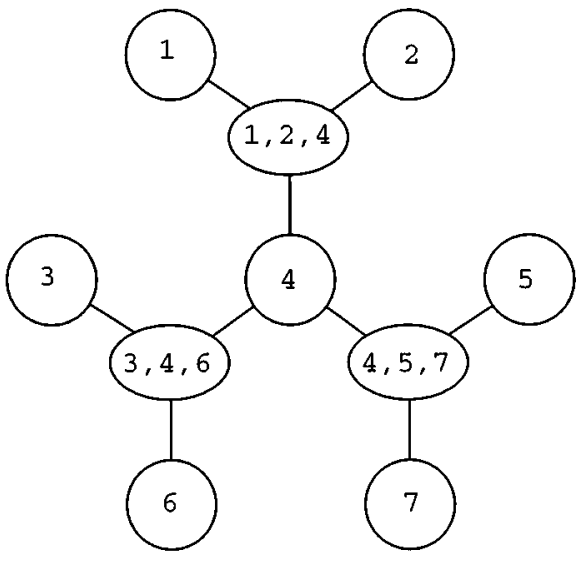

Fig. 14. A junction tree for Example 4.3.

yields the usual "fast" Hadamard transform. More generally, by extending the method in this example, it is possible to show that the FFT on any finite Abelian group, as described, e.g., in [8] or [31], can be derived from an application of the GDL. ${ }^{8}$

Example 4.3: Here we continue Example 2.3. In this case, the local domains can be organized as a junction tree. One such tree is shown in Fig. 14. It can be shown that the GDL, when applied to the junction tree of Fig. 14, yields the Gallager-Tanner-Wiberg algorithm [15], [34], [39] for decoding linear codes defined by cycle-free graphs. Indeed, Fig. 14 is identical to the "Tanner graph" cited by Wiberg [39] for decoding this particular code.

Example 4.4: Here we continue Example 2.4. The local domains can be arranged into a junction tree, as shown in Fig. 15. (In general, the junction tree has the same topology as DAG, if the DAG is cycle-free.) The GDL algorithm, when applied to the junction tree of Fig. 15, is equivalent to certain algorithms which are known in the artificial intelligence community for solving the probabilistic inference problem on Bayesian networks whose associated DAG's are cycle-free; in particular, Pearl's "belief propagation" algorithm [29], and the "probability propagation" algorithm of Shafer and Shenoy [33].

Example 4.5: Here we continue Example 2.5, the probabilistic state machine. In this case the local domains can be organized into a junction tree, as illustrated in Fig. 16 for the case $n=4$. The GDL algorithm, applied to the junction tree of Fig. 16, gives us essentially the BCJR [5] and Viterbi [37][11] algorithms, respectively. (For Viterbi's algorithm, we take the negative logarithm of the objective function in (2.5), and use the min-sum semiring, with a single target vertex, preferably the "last" $\left\{u_{i}\right\}$, which in Fig. 16 is $\left\{u_{3}\right\}$. For the BCJR algorithm, we use the objective function in (2.5) as it stands, and use the sum-product semiring, and evaluate the objective function at each of the vertices $\left\{u_{i}\right\}$, for $i=0, \cdots, n-1$. In both cases, the appropriate schedule is fully serial.)

\footnotetext{
${ }^{8}$ For this, see [1], where it is observed that the moral graph for the DFT over a finite Abelian group $G$ is triangulated if and only if $G$ is a cyclic group of prime-power order. In all other cases, it is necessary to triangulate the moral graph, as we have done in this example.
}

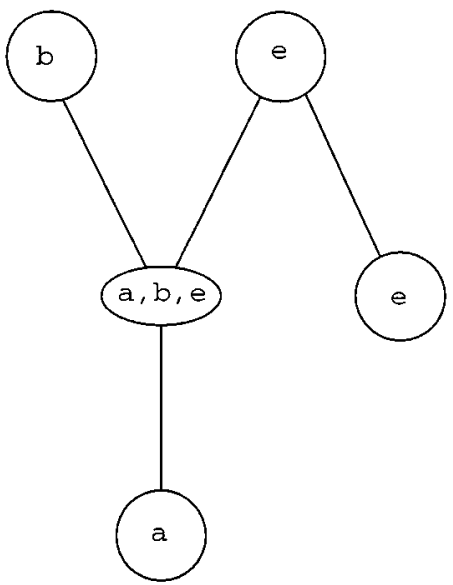

Fig. 15. A junction tree for Example 4.4. This figure should be compared to Fig. 1.

Example 4.6: Here we continue Example 2.6, the matrix multiplication problem. It is easy to see that for $n \geq 3$, there is no junction tree for the original set of local domains, because the corresponding moral graph is a cycle of length $n+1$. It is possible to show that for the product of $n$ matrices, there are

$$
\frac{1}{n}\left(\begin{array}{c}
2 n-2 \\
n-1
\end{array}\right)
$$

possible triangulations of the moral graph, which are in one-toone correspondence with the different ways to parenthesize the expression $M_{1} \cdots M_{n}$. For example, the parenthesization

$$
\left(\left(\cdots\left(M_{1} M_{2}\right) \cdots\right) M_{n-1}\right) M_{n}
$$

corresponds to the triangulation shown in Fig. 17.

Thus the problem of finding an optimal junction tree is identical to the problem of finding an optimal parenthesization. For example, in the case $n=3$, illustrated in Fig. 18, there are two different triangulations of the moral graph, which lead, via the techniques described in this section, to the two junction trees shown in the lower part of Fig. 18. With the top vertex as the target, the GDL applied to each of these trees computes the product $M_{1} M_{2} M_{3}$. The left junction tree corresponds to parenthesizing the product $M_{1} M_{2} M_{3}$ as $\left(M_{1} M_{2}\right) M_{3}$ and requires $2 q_{0} q_{1} q_{2}+2 q_{0} q_{2} q_{3}$ arithmetic operations, whereas the right junction tree corresponds to $M_{1}\left(M_{2} M_{3}\right)$ and requires $2 q_{1} q_{2} q_{3}+$ $2 q_{0} q_{1} q_{3}$ operations. Thus which tree one prefers depends on the relative size of the matrices. For example, if $q_{0}=10, q_{1}=100$, $q_{2}=5$, and $q_{3}=50$, the left junction tree requires 15000 operations and the right junction tree takes 150000 . (This example is taken from [9].)

As we discussed in Example 2.6, the matrix multiplication problem is equivalent to a trellis path problem. In particular, if the computations are in the min-sum semiring, the problem is that of finding the shortest paths in the trellis. If the moral graph is triangulated as shown in Fig. 17, the resulting junction tree yields an algorithm identical to Viterbi's algorithm. Thus Viterbi's algorithm can be viewed as an algorithm for multi- 


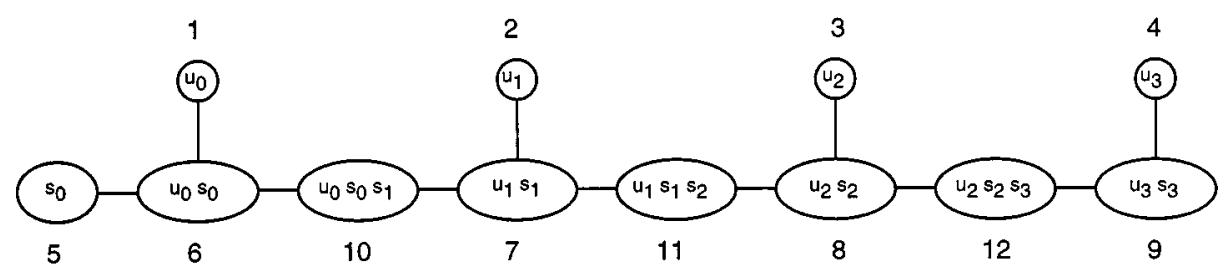

Fig. 16. A junction tree for the probabilistic state machine (illustrated for $n=4$ ).

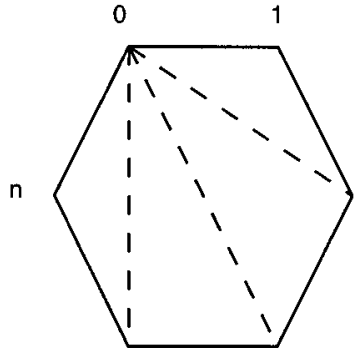

Fig. 17. The triangulation of the moral graph corresponding to the parenthesization $\left(\left(\cdots\left(M_{1} M_{2}\right) \cdots\right) M_{n-1}\right) M_{n}$.
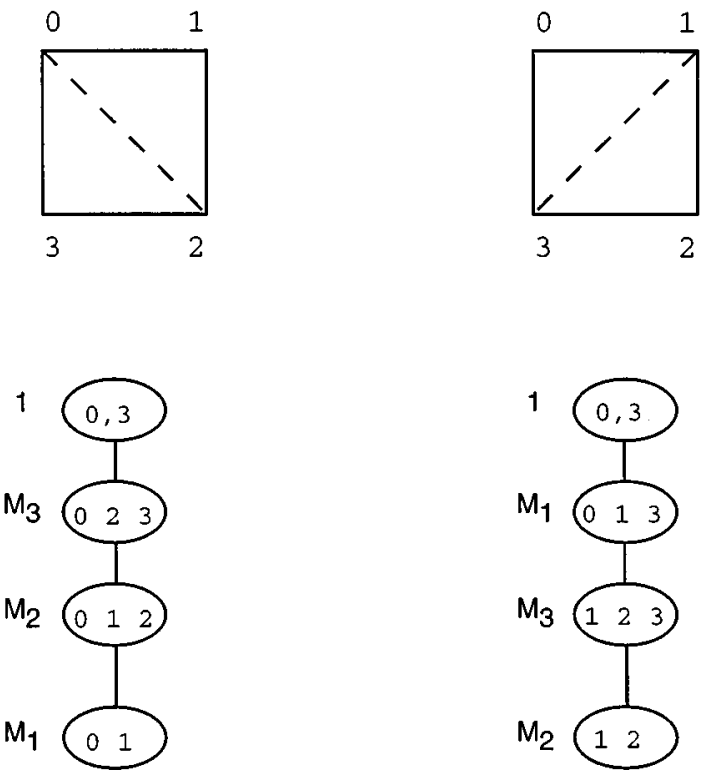

Fig. 18. The moral graph for Example 4.6, triangulated in two ways, and the corresponding junction trees. The left junction tree corresponds to the parenthesization $\left(M_{1} M_{2}\right) M_{3}$, and the one on the right corresponds to $M_{1}\left(M_{2} M_{3}\right)$

plying a chain of matrices in the min-sum semiring. (This connection is explored in more detail in [4].)

\section{COMPLEXITY OF THE GDL}

In this section we will provide complexity estimates for the serial versions of the GDL discussed in Section III. Here by complexity we mean the arithmetic complexity, i.e., the total number of (semiring) additions and/or multiplications required to compute the desired objective functions.
We begin by rewriting the message and state computation formulas (3.1) and (3.2), using slightly different notation. The message from vertex $v$ to vertex $w$ is defined as (cf. (3.1))

$$
\mu_{v, w}\left(x_{v \cap w}\right)=\sum_{x_{v \backslash w} \in A_{S(v) \backslash S(w)}} \alpha_{v}\left(x_{v}\right) \prod_{\substack{u \operatorname{adj} v \\ u \neq v}} \mu_{u, v}\left(x_{u \cap v}\right)
$$

and the state of vertex $v$ is defined as (cf. (3.2))

$$
\sigma_{v}\left(x_{v}\right)=\alpha_{v}\left(x_{v}\right) \prod_{u \operatorname{adj} v} \mu_{u, v}\left(x_{u \cap v}\right) .
$$

We first consider the single-vertex problem, supposing that $v_{0}$ is the target. For each $v \neq v_{0}$, there is exactly one edge directed from $v$ toward $v_{0}$. We suppose that this edge is $(v, w)$. To compute the message $\mu_{v, w}\left(x_{v \cap w}\right)$ as defined in (5.1) for a particular value of $x_{v \cap w}$ requires ${ }^{9}\left|A_{S(v) \backslash S(w)}\right|-1$ additions and $\left|A_{S(v) \backslash S(w)}\right|(d(v)-1)$ multiplications, where $d(v)$ is the degree of the vertex $v$. Using simplified but (we hope) self-explanatory notation we rewrite this as follows:

$$
\begin{aligned}
& q_{v \backslash w}-1 \text { additions, and } \\
& q_{v \backslash w} \cdot(d(v)-1) \text { multiplications. }
\end{aligned}
$$

But there are

$$
q_{v \cap w} \stackrel{\text { def }}{=}\left|A_{S(v) \cap S(w)}\right|
$$

possibilities for $x_{v \cap w}$, so the entire message $\mu_{v, w}\left(x_{v \cap w}\right)$ requires

$$
\begin{aligned}
& \left(q_{v \cap w}\right)\left(q_{v \backslash w}-1\right)=q_{v}-q_{v \cap w} \text { additions, and } \\
& \left(q_{v \cap w}\right) q_{v \backslash w} \cdot(d(v)-1)=(d(v)-1) q_{v} \text { multiplications. }
\end{aligned}
$$

The total number of arithmetic operations required to send messages toward $v_{0}$ along each of the edges of the tree is thus

$$
\begin{array}{ll}
\sum_{v \neq v_{0}}\left(q_{v}-q_{v} \cap w\right. & \text { additions } \\
\sum_{v \neq v_{0}}(d(v)-1) q_{v} & \text { multiplications. }
\end{array}
$$

When all the messages have been computed and transmitted, the algorithm terminates with the computation of the state at $v_{0}$, defined by (5.2). This state computation requires $d\left(v_{0}\right) q_{v_{0}}$ further multiplications, so that the total is

$$
\begin{aligned}
& \sum_{v \neq v_{0}}\left(q_{v}-q_{v \cap w}\right) \quad \text { additions } \\
& \sum_{v \neq v_{0}}(d(v)-1) q_{v}+d\left(v_{0}\right) q_{v_{0}} \quad \text { multiplications. }
\end{aligned}
$$

${ }^{9}$ Here we are assuming that the addition (multiplication) of $N$ elements of $S$ requires $N-1$ binary additions (multiplications). 
Thus the grand total number of additions and multiplications is

$$
\chi(T)=\sum_{v \in V} d(v) q_{v}-\sum_{e \in E} q_{e}
$$

where if $e=(v, w)$ is an edge, its "size" $q_{e}$ is defined to be $q_{v \cap n w}$.

Note that the formula in (5.3) gives the upper bound

$$
\chi(T) \leq \sum_{v \in V} d(v) q_{v}
$$

mentioned in Section III.

The formula in (5.3) can be rewritten in a useful alternative way, if we define the "complexity" of the edge $e=(v, w)$ as

$$
\chi(e)=q_{v}+q_{w}-q_{v \cap w} .
$$

With this definition, the formula in (5.3) becomes

$$
\chi(T)=\sum_{e \in E} \chi(e) .
$$

For example, for the junction tree of Fig. 4, there are four edges and

$$
\begin{aligned}
& \chi\left(v_{1}, v_{2}\right)=q_{1}+q_{1} q_{2}-q_{1}=q_{1} q_{2} \\
& \chi\left(v_{1}, v_{3}\right)=q_{1}+q_{1} q_{3}-q_{1}=q_{1} q_{3} \\
& \chi\left(v_{2}, v_{4}\right)=q_{1} q_{2}+q_{2}-q_{2}=q_{1} q_{2} \\
& \chi\left(v_{2}, v_{5}\right)=q_{1} q_{2}+q_{2} q_{4}-q_{2}
\end{aligned}
$$

so that

$$
\chi(T)=3 q_{1} q_{2}+q_{1} q_{3}+q_{2} q_{4}-q_{2} .
$$

We next briefly consider the all-vertices problem. Here a message must be sent over each edge, in both directions, and the state must be computed at each vertex. If this is done following the ideas above in the obvious way, the resulting complexity is $O\left(\sum_{v} d(v)^{2} q_{v}\right)$. However, we may reduce this by noticing that if $\left\{a_{1}, \cdots, a_{d}\right\}$ is a set of $d$ numbers, it is possible to compute all the $d$ products of $d-1$ of the $a_{i}$ 's with at most $3(d-2)$ multiplications, rather than the obvious $d(d-2)$. We do this by precomputing the quantities $b_{1}=a_{1}, b_{2}=b_{1} \cdot a_{2}=a_{1} a_{2}$, $\cdots, b_{d-1}=b_{d-2} \cdot a_{d-1}=a_{1} a_{2} \cdots a_{d-1}$, and $c_{d}=a_{d}$, $c_{d-1}=a_{d-1} c_{d}=a_{d-1} a_{d}, \cdots, c_{2}=a_{2} \cdot c_{3}=a_{2} a_{3} \cdots a_{d}$, using $2(d-2)$ multiplications. Then if $\hat{a}_{j}$ denotes the product of all the $a_{i}$ 's except for $a_{j}$, we have $\hat{a}_{1}=c_{2}, \hat{a}_{2}=b_{1} \cdot c_{3}, \cdots$, $\hat{a}_{d-1}=b_{d-2} \cdot c_{d}, \hat{a}_{d}=b_{d-1}$, using a further $d-2$ multiplications, for a total of $3(d-2)$. With one further multiplication $\left(b_{d-1} \cdot a_{d}\right)$, we can compute $b_{d}=a_{1} a_{2} \cdots a_{d} \cdot{ }^{10}$

Returning now to the serial implementation of the all-vertex GDL, each vertex must pass a message to each of its neighbors. Vertex $v$ will have $d(v)$ incoming messages, and (prior to marginalization) each outgoing message will be the product of $d(v)-1$ of these messages with the local kernel at $v$. For its own state computation, $v$ also needs the product of all $d(v)$ incoming messages with the local kernel. By the above argument, all this can be done with at most $3 d(v)$ multiplications for each of the $q_{v}$ values of the variables in the local domain at $v$. Thus the number of multiplications required is at most $3 \sum_{v} d(v) q_{v}$. The marginalizations during the message computations remain

\footnotetext{
${ }^{10}$ One of the referees has noted that the trick described in this paragraph is itself an application of the GDL; it has the same structure as the forward-backward algorithm applied to a trellis representing a repetition code of length $d$.
}

as in the single-vertex case, and, summed over all messages, require

$$
\sum_{v \in V} d(v) q_{v}-2 \sum_{e \in E} q_{e}
$$

additions. Thus the total number of arithmetic operations is no more than $4 \sum_{v} d(v) q_{v}$, which shows that the complexity of the all-vertices GDL is at worst a fixed constant times that of the single-vertex GDL. Therefore, we feel justified in defining the complexity of a junction tree, irrespective of which objective functions are sought, by (5.3) or (5.6). (In [23], the complexity of a similar, but not identical, algorithm was shown to be upper-bounded by $3 \sum_{v} q_{v}+M \max _{v} q_{v}$. This bound is strictly greater than the bound in (5.4).)

In Section IV, we saw that in many cases $w_{\max }=w^{*}$ and the LD graph has more than one maximal-weight spanning tree. In view of the results in this section, in such cases it is desirable to find the maximal-weight spanning tree with $\chi(T)$ as small as possible. It is easy to modify Prim's algorithm to do this. In Prim's algorithm, the basic step is to add to the growing tree a maximal-weight edge which does not form a cycle. If there are several choices with the same weight, choose one whose complexity, as defined by (5.5), is as small as possible. The tree that results is guaranteed to be a minimum-complexity junction tree [19]. In fact, we used this technique to find minimum-complexity junction trees in Examples 4.1, 4.3, 4.4, and 4.5.

We conclude this section with two examples which illustrate the difficulty of finding the minimum-complexity junction tree for a given marginalization problem. Consider first the local domains $\left\{x_{1}\right\},\left\{x_{2}\right\},\left\{x_{3}\right\}$, and $\left\{x_{1}, x_{2}, x_{3}\right\}$. There is a unique junction tree with these sets as vertex labels, shown in Fig. 19(a). By (5.3), the complexity of this junction tree is $3 q_{1} q_{2} q_{3}$. Now suppose we artificially enlarge the local domain $\left\{x_{2}\right\}$ to $\left\{x_{1}, x_{2}\right\}$. Then the modified set of local domains, viz., $\left\{x_{1}\right\},\left\{x_{1}, x_{2}\right\},\left\{x_{3}\right\}$, and $\left\{x_{1}, x_{2}, x_{3}\right\}$ can be organized into the junction tree shown in Fig. 19(b), whose complexity is $2 q_{1} q_{2} q_{3}+q_{1} q_{2}$, which is less than that of the original tree as long as $q_{3}>1$.

As the second example, we consider the domains $\left\{x_{1}, x_{4}\right\}$, $\left\{x_{2}, x_{5}\right\},\left\{x_{3}, x_{6}\right\}$, and $\left\{x_{1}, x_{2}, x_{3}\right\}$, which can be organized into a unique junction tree (Fig. 20(a)). If we adjoin the domain $\left\{x_{2}, x_{3}, x_{5}\right\}$, however, we can build a junction tree (Fig. 20(b)) whose complexity is lower than the original one, provided that $q_{1}$ is much larger than any of the other $q_{i}$ 's. (It is known that the problem of finding the "best" triangulation of a given graph is NP-complete [40], where "best" refers to having the minimum maximum clique size.)

\section{A BRIEF History OF THE GDL}

Important algorithms whose essential underlying idea is the exploitation of the distributive law to simplify a marginalization problem have been discovered many times in the past. Most of these algorithms fall into one of three broad categories: $d e$ coding algorithms, the "forward-backward algorithm," and artificial intelligence algorithms. In this section we will summarize these three parallel threads 


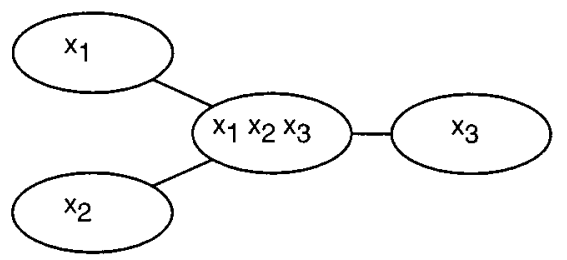

(a)

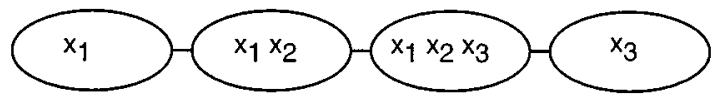

(b)

Fig. 19. Enlarging a local domain can lower the junction tree complexity.

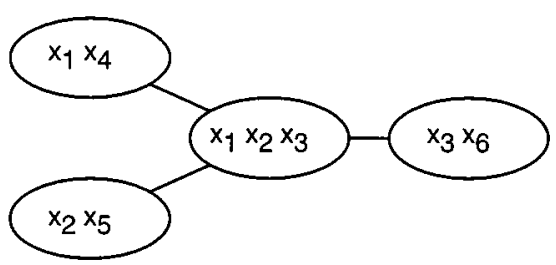

(a)

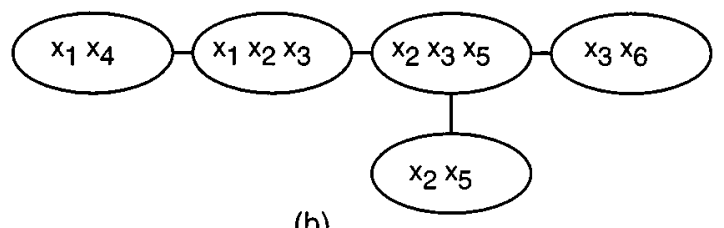

(b)

Fig. 20. Adding an extra local domain can lower the junction tree complexity.

\section{- Decoding Algorithms}

The earliest occurrence of a GDL-like algorithm that we are aware of is Gallager's 1962 algorithm for decoding low-density parity-check codes [15] [16]. Gallager was aware that his algorithm could be proved to be correct only when the underlying graphical structure had no cycles, but also noted that it gave good experimental results even when cycles were present. Gallager's work attracted little attention for 20 years, but in 1981 Tanner [34], realizing the importance of Gallager's work, made an important generalization of low-density parity-check codes, introduced the "Tanner graph" viewpoint, and recast Gallager's algorithm in explicit message-passing form. Tanner's work itself went relatively unnoticed until the 1996 thesis of Wiberg [39], which showed that the message-passing Tanner graph decoding algorithm could be used not only to describe Gallager's algorithm, but also Viterbi's and BCJR's. Wiberg too understood the importance of the cycle-free condition, but nevertheless observed that the turbo decoding algorithm was an instance of the Gallager-Tanner-Wiberg algorithm on a graphical structure with cycles. Wiberg explicitly considered both the sum-product and min-sum semirings, and speculated on the possibility of further generalizations to what he called "universal algebras" (our semirings).

In an independent series of developments, in 1967 Viterbi [37] invented his celebrated algorithm for maximum-likelihood decoding (minimizing sequence error probability) of convolutional codes. Seven years later (1974), Bahl, Cocke, Jelinek, and Raviv [5] published a "forward-backward" decoding algorithm (see next bullet) for minimizing the bit-error probability of convolutional codes. The close relationship between these two algorithms was immediately recognized by Forney [11]. Although these algorithms did not apparently lead anyone to discover a class of algorithms of GDL-like generality, with hindsight we can see that all the essential ideas were present.

\section{- The Forward-Backward Algorithm}

The forward-backward algorithm (also known as the $M$-step in the Baum-Welch algorithm) was invented in 1962 by Lloyd Welch, and seems to have first appeared in the unclassified literature in two independent 1966 publications [6], [7]. It appeared explicitly as an algorithm for tracking the states of a Markov chain in the early 1970's [5], [26] (see also the survey articles [30] and [32]). A similar algorithm (in min-sum form) appeared in a 1971 paper on equalization [35]. The algorithm was connected to the optimization literature in 1987 [36], where a semiring-type generalization was given.

\section{- Artificial Intelligence}

The relevant research in the artificial intelligence (AI) community began relatively late, but it has evolved quickly. The activity began in the 1980's with the work of Kim and Pearl [20] and Pearl [29]. Pearl's "belief propagation" algorithm, as it has come to be known, is a message-passing algorithm for solving the probabilistic inference problem on a Bayesian network whose DAG contains no (undirected) cycles. Soon afterwards, Lauritzen and Spiegelhalter [23] obtained an equivalent algorithm, and moreover generalized it to arbitrary DAG's by introducing the triangulation procedure. The notion of junction trees (under the name "Markov tree") was explicitly introduced by Shafer and Shenoy [33]. A recent book by Jensen [18] is a good introduction to most of this material. A recent unification of many of these concepts called "bucket elimination" appears in [10], and a recent paper by Lauritzen and Jensen [22] abstracts the MPF problem still further, so that the marginalization is done axiomatically, rather than by summation.

In any case, by early 1996, the relevance of these AI algorithms had become apparent to researchers in the information theory community [21] [28]. Conversely, the AI community has become excited by the developments in the information theory community [14] [38], which demonstrate that these algorithms can be successful on graphs with cycles. We discuss this is in the next section.

\section{ITERATIVE AND APPROXIMATE Versions OF THE GDL}

Although the GDL can be proved to be correct only when the local domains can be organized into a junction tree, the computations of the messages and states in (3.1) and (3.2) make 
sense whenever the local domains are organized as vertex labels on any kind of a connected graph, whether it is a junction tree or not. On such a junction graph, there is no notion of "termination," since messages may travel around the cycles indefinitely. Instead, one hopes that after sufficiently many messages have been passed, the states of the selected vertices will be approximately equal to the desired objective functions. This hope is based on a large body of experimental evidence, and some emerging theory.

\section{- Experimental Evidence}

It is now known that an application of the GDL, or one of its close relatives, to an appropriate junction graph with cycles, gives both the Gallager-Tanner-Wiberg algorithm for low-density parity-check codes [24], [25], [28],[39], the turbo decoding algorithm [21], [28], [39]. Both of these decoding algorithms have proved to be extraordinarily effective experimentally, despite the fact that there are as yet no general theorems that explain their behavior.

\section{- Emerging Theory: Single-Cycle Junction Graphs}

Recently, a number of authors [1]-[3], [12], [38], [39] have studied the behavior of the iterative GDL on junction graphs which have exactly one cycle. It seems fair to say that, at least for the sum-product and the min-sum semirings, the iterative GDL is fairly well understood in this case, and the results imply, for example, that iterative decoding is effective for most tail-biting codes. Although these results shed no direct light on the problem of the behavior of the GDL on multicycle junction graphs, like those associated with Gallager codes or turbo codes, this is nevertheless an encouraging step.

\section{APPENDIX A PROOF OF THE SCHEDULING THEOREM}

Summary: In this appendix, we will give a proof of the Scheduling Theorem 3.1, which will prove the correctness of the GDL. The key to the proof is Corollary A.4, which tells us that at every stage of the algorithm, the state at a given vertex is the appropriately marginalized product of a subset of the local kernels. Informally, we say that at time $t$, the state at vertex $v$ is the marginalized product of the local kernels which are currently "known" to $v$. Given this result, the remaining problem is to understand how knowledge of the local kernels is disseminated to the vertices of the junction tree under a given schedule. As we shall see, this "knowledge dissemination" can be described recursively as follows:

- Rule (1): Initially $(t=0)$, each vertex $v_{i}$ knows only its own local kernel $\alpha_{i}$.

- Rule (2): If a directed edge $\left(v_{i}, v_{j}\right)$ is activated at time $t$, i.e., if $\left(v_{i}, v_{j}\right) \in E_{t}$, then vertex $v_{j}$ learns all the local kernels known to $v_{i}$ at time $t-1$.

The proof of Theorem 3.1 then follows quickly from these rules.

We begin by introducing some notation. Let $f\left(x_{S}\right)$ be a function of the variable list $x_{S}$, and let $T$ be an arbitrary subset of

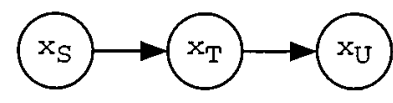

Fig. 21. A junction tree for Lemma A.1.

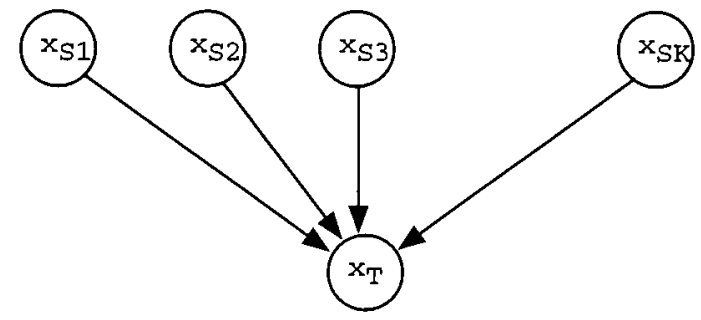

Fig. 22. A junction tree for Lemma A.2.

$\{1, \cdots, n\}$. We denote by $\left[f\left(x_{S}\right)\right]^{T}$ the function of the variable list $x_{S \cap T}$ obtained by "marginalizing out" the variables in $f$ which are not in $T$ :

$$
\left[f\left(x_{S}\right)\right]^{T} \stackrel{\text { def }}{=} \sum_{x_{S \backslash T}} f\left(x_{S}\right) .
$$

Lemma A.1: If $S \cap U \subseteq T$, then

$$
\left[\left[f\left(x_{S}\right)\right]^{T}\right]^{U}=\left[f\left(x_{S}\right)\right]^{U} .
$$

Proof: (Note first that Lemma A.1 is a special case of the single-vertex GDL, with the following local domains and kernels.

$\begin{array}{lcc} & \text { local domain } & \text { local kernel } \\ \text { 1. } & \left\{x_{S}\right\} & f\left(x_{S}\right) \\ 2 . & \left\{x_{T}\right\} & 1 \\ 3 . & \left\{x_{U}\right\} & 1 .\end{array}$

The appropriate junction tree is shown in Fig. 21.)

To see that the assertion is true, note that the variables not marginalized out in the function $\left[\left[f\left(x_{S}\right)\right]^{T}\right]^{U}$ are those indexed by $S \cap T \cap U$. The variables not marginalized out in $\left[f\left(x_{S}\right)\right]^{U}$ are those indexed by $S \cap U$. But by the hypothesis $S \cap U \subseteq T$, these two sets are equal.

Lemma A.2: Let $f_{i}\left(x_{S_{i}}\right)$, for $i=1, \cdots, K$ be local kernels, and consider the MPF problem of computing

$$
\beta\left(x_{\left(S_{1} \cup \cdots S_{K}\right) \cap T}\right) \stackrel{\text { def }}{=}\left[\prod_{i=1}^{K} f_{i}\left(x_{S_{i}}\right)\right]^{T} .
$$

If no variable which is marginalized out in (A.1) occurs in more than one local kernel, i.e., if $S_{i} \cap S_{j} \subseteq T$ for $i \neq j$, then

$$
\beta\left(x_{\left(S_{1} \cup \cdots S_{K}\right) \cap T}\right)=\prod_{i=1}^{K}\left[f_{i}\left(x_{S_{i}}\right)\right]^{T} .
$$

Proof: (Lemma A.2 is also a special case of the singlevertex GDL, with the following local domains and kernels:

$$
\begin{array}{ccc} 
& \text { local domain } & \text { local kernel } \\
1 & \left\{x_{S_{1}}\right\} & f_{1}\left(x_{S_{1}}\right) \\
\vdots & \vdots & \vdots \\
K & \left\{x_{S_{K}}\right\} & f_{K}\left(x_{S_{K}}\right) \\
K+1 & \left\{x_{T}\right\} & 1
\end{array}
$$

The appropriate junction tree is shown in Fig. 22.) 
In any case, Lemma A. 2 is a simple consequence of the distributive law: Since each variable being marginalized out in (A.1) occurs in at most one local kernel, it is allowable to take the other local kernels out of the sum by distributivity. As an example, we have

$$
\begin{aligned}
\sum_{x_{1}, x_{3}, x_{4}} & f_{1}\left(x_{1}, x_{2}\right) f_{2}\left(x_{2}, x_{3}\right) f_{3}\left(x_{2}, x_{4}\right) \\
= & \sum_{x_{1}} f_{1}\left(x_{1}, x_{2}\right) \sum_{x_{3}} f_{2}\left(x_{2}, x_{3}\right) \sum_{x_{4}} f_{3}\left(x_{2}, x_{4}\right) .
\end{aligned}
$$

Now we are ready to consider the dynamics of the GDL. Consider an edge: $e_{i j}: v_{i} \rightarrow v_{j}$. Removing $e_{i j}$ from the junction tree $T$ breaks it into two components, $T_{i j}$ and $T_{j i}$ (see Fig. 23). For future reference, we denote the vertex set of $T_{i j}$ by $V_{i j}$, and the edge set by $E_{i j}$.

Since $e_{i j}$ is on the unique path between any vertex in $T_{i j}$ and any vertex in $T_{j i}$, it follows from the junction tree property that any variable which occurs in a vertex in both components must occur in both $v_{i}$ and $v_{j}$. Thus the message $\mu_{i, j}$, which may be viewed as a message from $T_{i j}$ to $T_{j i}$, is a function of exactly those variables which occur in both components.

In what follows, for each index $i=1, \cdots, M$ we define

$$
N_{i}=\left\{k:\left(v_{k}, v_{i}\right) \in E\right\}
$$

and for each pair of indices $(i, j)$ such that $\left(v_{i}, v_{j}\right) \in E$, we define

$$
N_{i, j}=\left\{k:\left(v_{k}, v_{i}\right) \in E, k \neq j\right\} .
$$

In words, $N_{i}$ represents the (indices of) the neighbors of $v_{i}$, and $N_{i, j}$ represents the (indices of) the neighbors of $v_{i}$ other than $v_{j}$.

Now let $\mathcal{E}=\left(E_{1}, E_{2}, \cdots, E_{N}\right)$ be a schedule for a junction tree, as defined in Section III, i.e., a finite list of subsets of $E$, and let $\mu_{i, j}(t)$ be the value of the message $\mu_{i, j}$ after the $t$ th round of $\mathcal{E}$.

Theorem A.3: The message $\mu_{i, j}(t)$ is the product of a subset of the local kernels in $T_{i j}$, with the variables that do not occur in $T_{j i}$ marginalized out. Specifically, we have

$$
\mu_{i, j}(t)=\left[\prod_{k \in K_{i, j}(t)} \alpha_{k}\right]^{S_{j}}
$$

where $K_{i, j}(t)$ is a subset of $V_{i j}$, the vertex set of $T_{i j}$. The sets $K_{i, j}(t)$ are defined inductively as follows:

$$
\begin{aligned}
K_{i, j}(0) & =\emptyset, \quad \text { and for } t \geq 1 \\
K_{i, j}(t) & = \begin{cases}K_{i, j}(t-1), & \text { if } e_{i j} \notin E_{t} \\
\{i\} \bigcup_{\ell \in N_{i, j}} K_{\ell, i}(t-1), & \text { if } e_{i j} \in E_{t} .\end{cases}
\end{aligned}
$$

Proof: We use induction on $t$, the case $t=0$ being simply a restatement of the initialization rule $\mu_{i, j}=1$. Assuming the

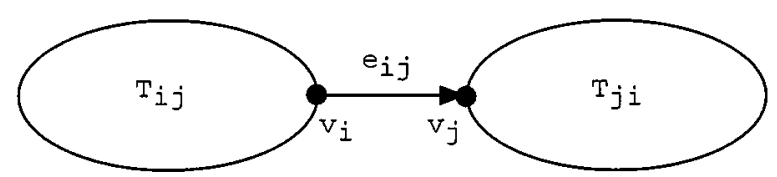

Fig. 23. Deleting the edge $e_{i j}$ breaks the junction tree into two components.

theorem proved for $t-1$, we assume $e_{i, j}$ is updated in the $t$ th round, and consider $\mu_{i, j}(t)$ :

$$
\begin{aligned}
\mu_{i, j}(t) & =\left[\alpha_{i} \prod_{\ell \in N_{i, j}} \mu_{\ell, i}(t-1)\right]^{S_{j}} \text { by }(3.1) \\
& =\left[\alpha_{i} \prod_{\ell \in N_{i, j}}\left[\prod_{k \in K_{\ell, i}(t-1)} \alpha_{k}\right]^{S_{i}}\right]^{S_{j}} \text { by induction. }
\end{aligned}
$$

Any variable that occurs in two different messages $\mu_{\ell_{1}, i}(t-1)$ and $\mu_{\ell_{2}, i}(t-1)$ must also, by the junction tree property, occur in $\alpha_{i}$, so we may apply Lemma A.2 to rewrite the last line as

$$
=\left[\left[\alpha_{i} \prod_{\ell \in N_{i, j}} \prod_{k \in K_{\ell, i}(t-1)} \alpha_{k}\right]^{S_{i}}\right]^{S_{j}} .
$$

Since a variable that occurs in one of the kernels in the above equation and also in $v_{j}$ must, by the junction tree property, also occur in $v_{i}$, it follows from Lemma A.1 that this last expression can be simplified to

$$
\left[\alpha_{i} \prod_{\ell \in N_{i, j}} \prod_{k \in K_{\ell, i}(t-1)} \alpha_{k}\right]^{S_{j}}=\left[\prod_{k \in K_{i, j}(t)} \alpha_{k}\right]^{S_{j}}
$$

the last equality because of the definition (A.3).

Corollary A.4: For all $t \geq 0$, the state $\sigma_{i}(t)$ has the value

$$
\sigma_{i}(t)=\left[\prod_{k \in J_{i}(t)} \alpha_{k}\right]^{S_{i}}
$$

where the set $J_{i}(t)$ is defined by

$$
J_{i}(t)=\{i\} \bigcup_{j \in N_{i}} K_{j, i}(t)
$$

Proof: By definition (3.2)

$$
\begin{aligned}
\sigma_{i}(t) & =\alpha_{i} \prod_{j \in N_{i}} \mu_{j, i}(t) \\
& =\alpha_{i}^{S} \prod_{j \in N_{i}} \mu_{j, i}(t) .
\end{aligned}
$$


(We know that $\alpha_{i}=\alpha_{i}^{S_{i}}$, since the kernel $\alpha_{i}$ is by definition a function only of the variables involved in the local domain $S_{i}$.) By Theorem A.3, this can be written as

$$
\sigma_{i}(t)=\alpha_{i}^{S_{i}} \prod_{j \in N_{i}}\left[\prod_{k \in K_{j, i}(t)} \alpha_{k}\right]^{S_{i}}
$$

But by the junction tree property, any variable that occurs in two of the bracketed terms must also occur in $\alpha_{i}$, so that by Lemma A. 2

$$
\begin{aligned}
\sigma_{i}(t) & =\left[\alpha_{i} \prod_{j \in N_{i}} \prod_{k \in K_{j, i}(t)} \alpha_{k}\right]^{S_{i}} \\
& =\left[\prod_{k \in J_{i}(t)} \alpha_{k}\right]^{S_{i}} \text { by the definition (A.5). }
\end{aligned}
$$

Theorem A.3 tells us that at time $t$, the message from $v_{i}$ to $v_{j}$ is the appropriately marginalized product of a subset of the local kernels, viz., $\left\{\alpha_{k}: k \in K_{i, j}(t)\right\}$, and Corollary A.4 tells us that at time $t$, the state of vertex $v_{i}$ is the appropriately marginalized product of a subset of the local kernels, viz., $\left\{\alpha_{k}: k \in J_{i}(t)\right\}$, which we think of as the subset of local kernels which are "known" to $v_{i}$ at time $t$. Given these results, the remaining problem is to understand how knowledge of the local kernels is disseminated to the vertices of the junction tree under a given schedule. A study of (A.5), which gives the relationship between what is known at the vertex $v_{i}$ and what is known by the incoming edges, together with the message update rules in (A.3), provides a nice recursive description of exactly how this information is disseminated:

- Rule (1): Initially $(t=0)$, each vertex $v_{i}$ knows only its own local kernel $\alpha_{i}$.

- Rule (2): If a directed edge $\left(v_{i}, v_{j}\right)$ is activated at time $t$, i.e., if $\left(v_{i}, v_{j}\right) \in E_{t}$, then vertex $v_{j}$ learns all the local kernels previously known to $v_{i}$ at time $t-1$.

We shall now use these rules to prove Theorem 3.1.

Theorem 3.1 asserts that $v_{i}$ knows each of the $M$ local kernels $\alpha_{1}, \cdots, \alpha_{M}$ at time $t=N$ if and only if there is a path in the message trellis from $v_{j}(0)$ to $v_{i}(N)$, for all $j=1,2, \cdots, M$. We will now prove the slightly more general statement that $v_{i}$ knows $\alpha_{j}$ at time $t=N$ if and only if there is a path in the message trellis from $v_{j}(0)$ to $v_{i}(N)$.

To this end, let us first show that if $v_{i}$ knows $\alpha_{j}$ at $t=N$, then there must be a path in the message trellis from $v_{j}(0)$ to $v_{i}(N)$. Because we are in a tree, there is a unique path from $v_{j}$ to $v_{i}$, say

$$
\mathbb{P}_{j, i}=\left[v_{k_{0}}, v_{k_{1}}, \cdots, v_{k_{L-1}}, v_{k_{L}}\right]
$$

where $k_{0}=j$ and $k_{L}=i$. Denote by $t_{\ell}$ the first (smallest) time index for which $v_{k_{\ell}}$ knows $\alpha_{j}$. Then by Rule 2 and an easy induction argument, we have

$$
1 \leq t_{1}<t_{2}<\cdots<t_{L} \leq N
$$

(In words, knowledge of $\alpha_{j}$ must pass sequentially to $v_{i}$ through the vertices of the path $\mathbb{P}_{j, i}$.) In view of (A.6), we have the following path from $v_{j}(0)=v_{k_{0}}(0)$ to $v_{i}(N)=v_{k_{L}}(N)$ in the message trellis from $v_{j}(0)$ to $v_{i}(N)$ :

$$
\begin{aligned}
& {\left[v_{k_{0}}(0), \cdots, v_{k_{0}}\left(t_{1}-1\right) ; v_{k_{1}}\left(t_{1}\right), \cdots, v_{k_{1}}\left(t_{2}-1\right)\right.} \\
& \left.\quad \cdots ; v_{k_{L}}\left(t_{L}\right), \cdots, v_{k_{L}}(N)\right] .
\end{aligned}
$$

Conversely, suppose there is a path from $v_{j}(0)$ to $v_{i}(N)$ in the message trellis. Then since apart from "pauses" at a given vertex, this path in the message trellis must be the unique path $\mathbb{P}_{i, j}$ from $v_{j}$ to $v_{i}$, Rule 2 implies that knowledge of the kernel $\alpha_{j}$ sequentially passes through the vertices on the path $\mathbb{P}_{i, j}$, finally reaching $v_{i}$ at time $N$.

This completes the proof of Theorem 3.1.

\section{REFERENCES}

[1] S. M. Aji, "Graphical models and iterative decoding," Ph.D. dissertation, Cal. Inst. Technol., Pasadena, CA, 1999.

[2] S. M. Aji, G. B. Horn, and R. J. McEliece, "On the convergence of iterative decoding on graphs with a single cycle," in Proc. 32nd Conf. Information Sciences and Systems, Princeton, NJ, Mar. 1998.

[3] S. M. Aji, G. B. Horn, R. J. McEliece, and M. Xu, "Iterative min-sum decoding of tail-biting codes," in Proc. IEEE Information Theory Workshop, Killarney, Ireland, June 1998, pp. 68-69.

[4] S. M. Aji, R. J. McEliece, and M. Xu, "Viterbi's algorithm and matrix multiplication," in Proc. 33rd Conf. Information Sciences and Systems, Baltimore, MD, Mar. 1999.

[5] L. R. Bahl, J. Cocke, F. Jelinek, and J. Raviv, "Optimal decoding of linear codes for minimizing symbol error rate," IEEE Trans. Inform. Theory, vol. IT-20, pp. 284-287, Mar. 1974.

[6] L. E. Baum and T. Petrie, "Statistical inference for probabilistic functions of finite-state Markov chains," Ann. Math. Stat, vol. 37, pp. 1559-1563, 1966.

[7] R. W. Chang and J. C. Hancock, "On receiver structures for channels having memory," IEEE Trans. Inform. Theory, vol. IT-12, pp. 463-468, Oct. 1966.

[8] J. W. Cooley and J. W. Tukey, "An algorithm for the machine calculation of complex Fourier series," Math. Comp., vol. 19, p. 297, Apr. 1965.

[9] T. H. Cormen, C. E. Leiserson, and R. L. Rivest, Introduction to Algorithms. Cambridge, MA: MIT-McGraw-Hill, 1990.

[10] R. Dechter, "Bucket elimination: A unifying framework for probabilistic inference," Artificial Intell., vol. 113, pp. 41-85, 1999.

[11] G. D. Forney Jr., "The Viterbi algorithm," Proc. IEEE, vol. 61, pp. 268-278, Mar. 1973.

[12] G. D. Forney Jr., F. R. Kschischang, and B. Marcus, "Iterative decoding of tail-biting trellises," in IEEE Information Theory Workshop, San Diego, CA, Feb. 1998, pp. 11-12.

[13] B. J. Frey, "Bayesian networks for pattern classification, data compression, and channel coding," Ph.D. dissertation, Univ. Toronto, Toronto, ON, Canada, 1997

[14] B. J. Frey and D. J. C. MacKay, "A revolution: Belief propagation in graphs with cycles," in Advances in Neural Information Processing Systems, M. I. Jordan, M. I. Kearns, and S. A. Solla, Eds. Cambridge, MA: MIT Press, 1998, pp. 470-485.

[15] R. G. Gallager, "Low-density parity-check codes," IRE Trans. Inform. Theory, vol. IT-8, pp. 21-28, Jan. 1962.

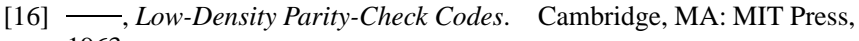
1963.

[17] R. C. Gonzalez and R. E. Woods, Digital Image Processing. Reading, MA: Addison-Wesley, 1992.

[18] F. V. Jensen, An Introduction to Bayesian Networks. New York: Springer-Verlag, 1996.

[19] F. V. Jensen and F. Jensen, "Optimal junction trees," in Proc. 10th Conf. Uncertainty in Artificial Intelligence, R. L. de Mantaras and D. Poole, Eds. San Francisco, CA, 1994, pp. 360-366.

[20] J. H. Kim and J. Pearl, "A computational model for causal and diagnostic reasoning," in Proc. 8th Int. Joint Conf. Artificial Intelligence, 1983, pp. 190-193. 
[21] F. R. Kschischang and B. J. Frey, "Iterative decoding of compound codes by probability propagation in graphical models," IEEE J. Select. Areas Commun., vol. 16, pp. 219-230, Feb. 1998.

[22] S. L. Lauritzen and F. V. Jensen, "Local computation with valuations from a commutative semigroup," Ann. Math. AI, vol. 21, no. 1, pp. 51-69, 1997.

[23] S. L. Lauritzen and D. J. Spiegelhalter, "Local computation with probabilities on graphical structures and their application to expert systems," J. Roy. Statist. Soc. B, pp. 157-224, 1988.

[24] D. J. C. MacKay and R. M. Neal, "Good codes based on very sparse matrices," in Cryptography and Coding, 5th IMA Conf., ser. Springer Lecture Notes in Computer Science No. 1025. Berlin, Germany: SpringerVerlag, 1995, pp. 100-111.

[25] — "Near Shannon limit performance of low density parity-check codes," Electron. Lett., vol. 33, pp. 457-458, 1996.

[26] P. L. McAdam, L. R. Welch, and C. L. Weber, "M.A.P. bit decoding of convolutional codes," in Proc. 1972 IEEE Int. Symp. Information Theory, Asilomar, CA, Jan. 1972, p. 91.

[27] R. J. McEliece, R. B. Ash, and C. Ash, Introduction to Discrete Mathematics. New York: Random House, 1989.

[28] R. J. McEliece, D. J. C. MacKay, and J. -F. Cheng, "Turbo decoding as an instance of Pearl's belief propagation algorithm," IEEE J. Select. Areas Comm., vol. 16, pp. 140-152, Feb. 1998.

[29] J. Pearl, Probabilistic Reasoning in Intelligent Systems. San Mateo, CA: Morgan Kaufmann, 1988.

[30] A. M. Poritz, "Hidden Markov models: A guided tour," in Proc. 1988 IEEE Conf. Acoustics, Speech, and Signal Processing, vol. 1, pp. 7-13.
[31] E. C. Posner, "Combinatorial structures in planetary reconnaissance," in Error Correcting Codes, H. B. Mann, Ed. New York: Wiley, 1968.

[32] L. Rabiner, "A tutorial on hidden Markov models and selected applications in speech recognition," Proc. IEEE, vol. 77, pp. 257-285, 1989.

[33] G. R. Shafer and P. P. Shenoy, "Probability propagation," Ann. Math. Art. Intel., vol. 2, pp. 327-352, 1990.

[34] R. M. Tanner, "A recursive approach to low complexity codes," IEEE Trans. Inform. Theory, vol. IT-27, pp. 533-547, Sep. 1981

[35] G. Ungerboeck, "Nonlinear equalization of binary signals in Gaussian noise," IEEE Trans. Commun. Technol., vol. COM-19, pp. 1128-1137, Dec. 1971.

[36] S. Verdú and V. Poor, "Abstract dynamic programming models under commutativity conditions," SIAM J. Contr. Optimiz., vol. 25, pp. 990-1006, July 1987.

[37] A. J. Viterbi, "Error bounds for convolutional codes and an asymptotically optimum decoding algorithm," IEEE Trans. Inform. Theory, vol IT-13, pp. 260-269, Apr. 1967.

[38] Y. Weiss, "Correctness of local probability propagation in graphical models with loops," Neural Comput., vol. 12, pp. 1-41, 2000.

[39] N. Wiberg, "Codes and decoding on general graphs ," dissertation no. 440, Linkoping Studies in Science and Technology, Linkoping, Sweden, 1996.

[40] M. Yannakakis, "Computing the minimum fill-in is NP-complete," SIAM J. Alg. Discr. Methods, vol. 2, pp. 77-79, 1981.

[41] F. R. Kschischang, B. J. Frey, and H.-A. Loeliger, "Factor graphs and the sum-product algorithm," IEEE Trans. Inform. Theory, submitted for publication. 